\title{
Independent Vector Analysis: Identification Conditions and Performance Bounds
}

\author{
Matthew Anderson, Geng-Shen Fu, Ronald Phlypo, and Tülay Adalı
}

\begin{abstract}
Recently, an extension of independent component analysis (ICA) from one to multiple datasets, termed independent vector analysis (IVA), has been the subject of significant research interest.|IVA has also been shown to be a generalization of Hotelling's canonical correlation analysis. In this paper, we provide the identification conditions for a general IVA formulation, which accounts for linear, nonlinear, and sample-to-sample dependencies. The identification conditions are a generalization of previous results for ICA and for IVA when samples are independently and identically distributed Furthermore, a principal aim of IVA is the identification of dependent sources between datasets. Thus, we provide the additional conditions for when the arbitrary ordering of the sources within each dataset is common. Performance bounds in terms of the Cramér-Rao lower bound are also provided for the demixing matrices and interference to source ratio The performance of two IVA algorithms are compared to the theoretical bounds.
\end{abstract}

\section{Motivation AND INTRODUCTION}

Blind source separation (BSS) problems have been well studied and many algorithms have been developed and successfully applied in a vast array of applications [1], [2]. A generalization of the BSS problem to multiple datasets, termed joint blind source separation (JBSS), has been introduced recently [3], [4]. The recent interest in JBSS is motivated by various application domains such as when analyzing multisubject datasets in biomedical studies using functional magnetic resonance imaging or electroencephalography data [3], [4] or when solving the convolutive independent component analysis (ICA) problem in the frequency domain using multiple frequency bins [5]. Interestingly, several algorithms developed prior to the development of the BSS concept are capable of achieving JBBSS [6], [7]. Thus, a much larger set of applications than the examples above are well treated using the JBSS formulation.

One particular formulation of JBSS has been termed independent vector analysis (IVA), The formulation of IVA is an extension of the (linear, instantaneous) ICA model. IVA assumes a source within one dataset is dependent on at most one source in another dataset while sources within a dataset are mutually independent (as in ICA). Thus, IVA reduces to performing ICA individually on each dataset when sources possess no dependence across datasets. Of particular interest here is to determine the conditions when IVA is identifiable. For a real-valued single dataset problem, independent sources can be 'blindly' identified up to a permutation and scaling ambiguity as long as no two sources are Gaussian with proportional sample-to-sample correlation matrices [2, Chapter 4]. The IVA framework has been shown to possess an additional type of diversity which can be exploited for identifying sources that cannot be identified by [CA] [8].

In this paper, a general framework for IVA is presented. By 'general' we mean an IVA formulation that accounts for dependency between samples, i.e., when the samples are not independently and identically distributed (iid) Prior to introducing this IVA formulation in Section IV, we give a review of existing IVA algorithms in Section II and define our mathematical conventions and notations in Section III. Naturally, IVA can be achieved by maximizing the likelihood function, which is shown in Section V to be the same in practice as minimizing the entropy rate (subject to a regularity term). The likelihood function has an associated Fisher information matrix (FIM) of a form that we describe in Section VI. The FIM is used in deriving the identification conditions and source separation performance bounds in Sections VII and VIII, respectively. The IVA identification conditions and performance bounds are generalizations of the results for ICA (of a single dataset). The IVA case when samples are iid is shown to have a performance bound that can be expressed compactly for the very large class of multivariate elliptical distributions. In Section IX, the performance bounds are compared to the performance achieved by two previously published algorithms for IVA. In the last section, we discuss directions for future work.

\section{ReVIEW OF Existing IVA Algorithms}

As mentioned previously, the origins of algorithms that can be used for IVA date back to pre-ICA times. In fact, classical canonical correlation analysis (CCA) [9] achieves IVA for linearly dependent sources in analysis of two datasets. The formulation of CCA can be shown to serve as a basis for all IVA algorithms reviewed here. This is because CCA can be derived from two different, but related principles; maximum likelihood and eigenanalysis (diagonalization). Here, we choose to separate the approaches into three classes for our review based on the source diversity exploited to achieve JBSS. It will be shown that each type of diversity can be utilized—independent of the other two- to achieve IVA. 


\section{A. Linear dependence}

The first class is applicable to problems in which the sources are assumed to have linear dependence across datasets, but are linearly independent within datasets. The earliest approaches to extending CCA beyond two datasets are summarized in [6] and has been termed multiset canonical correlation analysis (MCCA) in [4]. The approaches within MCCA use cost functions based on second-order statistics that result in JBSS solutions that can be widely applied. Another approach to JBSS for linearly dependent sources can be derived using equivalently maximum likelihood or minimization of mutual information and results in IVA with multivariate Gaussian distribution model [10], [11].

Since CCA can be achieved using generalized eigenvalue decomposition, it can also be posed as a diagonalization problem, which can be readily extended to achieve IVA using 'generalized joint diagonalization' [12]. For IVA of linearly dependent sources the covariance and cross-covariance matrices among the estimated sources in each dataset can be diagonalized as in [12], [13].

\section{B. Nonlinear dependence}

When the sources possess nonlinear dependence across the datasets then higher-order statistics should be utilized either explicitly or implicitly. The extension of CCA to nonlinear dependence measures for two datasets dates back to at least 1976 [7]. Extensions to multiple datasets is given in [14]. These early works are summarized in [15].

Another extension for nonlinear CCA of two datasets uses nonparametric univariate and bivariate density estimators in order to maximize the mutual information between two canonical correlation variates [16]. Kernels have also been used to transform the random vectors into a 'feature-space' where linear CCA is then applied [17], [18]. A different type of transformation is proposed in [19]. Here measure transform functions are specified for transforming joint probability measures to identify nonlinearly dependent sources. To use either the kernel or measure transform approaches, one must determine the appropriate transform and transform parameters to achieve JBSS for the problem at hand.

[VA also provides a framework for exploiting nonlinear dependencies. [VA] as first introduced in [20], [21] and in the similar work of [22], extends ICA to multiple datasets so as to solve the permutation ambiguity problem associated with frequency domain ICA [23]. The nonlinear dependencies can be accounted for within the IVA framework by considering non-Gaussian sources. For example, in [20], [21], a nonlinear score function consistent with the second-order uncorrelated multivariate Laplacian distribution is used.

As is the case for linear dependence, diagonalization methods for IVA of nonlinearly dependent sources can be utilized. Specifically, demixing matrices that diagonalize the higher-order statistics (i.e., cumulants of order higher than two) associated with the estimated sources are found [12], [13], [24].

\section{Sample-to-sample dependence}

Naturally for IVA, as for ICA algorithms can be developed to exploit sample-to-sample dependence. A generalization of joint diagonalization provides such a solution by sampling the vector autocorrelation function at different time lags and finding demixing matrices which minimize correlation between the sources for all time lags, see, e.g., [12], [13].

\section{Mathematical Preliminaries}

For this paper, the domains are restricted to the sets of real $(\mathbb{R})$ and nonnegative natural $(\mathbb{N})$ numbers. Matrices and vectors from each domain are indicated by $\mathbb{R}^{M \times N}, \mathbb{R}^{M}, \mathbb{N}^{M \times N}$, and $\mathbb{N}^{M}$, respectively. Scalar, (column) vector, and matrix quantities are denoted as lower-case light face, lower-case bold face, and upper-case bold face, respectively. The $m$ th element of a vector $\mathbf{v},[\mathbf{v}]_{m}$, and an element in the $m$ th row and $n$th column of a matrix $\mathbf{A},[\mathbf{A}]_{m, n}$, are often denoted $v_{m}$ and $a_{m, n}$, respectively.

The Kronecker delta, $\delta_{m, n}$, is one when $m=n$ and zero otherwise. The standard basis vector, $\mathbf{e}_{n}$, is the the $n$th column of identity matrix, $\mathbf{I}_{N} \in \mathbb{R}^{N \times N}$. The $\mathbf{0}$ and $\mathbf{1}$ denote matrices (or vectors) with all entries of zeros and ones, respectively, where the dimensions of the matrices are either known from the context or indicated by an additional subscript.

The superscript $T$ denotes the matrix transpose. The element-wise (Hadamard) product, element-wise division, and Kronecker products are denoted by $\mathbf{A} \circ \mathbf{B}, \mathbf{A} \oslash \mathbf{B}$, and $\mathbf{A} \otimes \mathbf{B}$, respectively. We use vec $(\mathbf{A}) \in \mathbb{R}^{M N}=\sum_{n=1}^{N} \mathbf{e}_{n} \otimes\left(\mathbf{A} \mathbf{e}_{n}\right)$, where $\mathbf{e}_{n} \in \mathbb{R}^{N}$, to compactly denote the the stacking of the columns of $\mathbf{A} \in \mathbb{R}^{M \times N}$. Additionally, if a subset of the rows in $\mathbf{A}$ are listed in the vector $\boldsymbol{\alpha}=\left[\alpha_{1}, \ldots, \alpha_{d}\right]^{\top} \in \mathbb{N}^{d}$, where $0 \leq d \leq M$ with a corresponding indexing matrix $\mathbf{E}_{[\boldsymbol{\alpha}]}=$ $\left[\mathbf{e}_{\alpha_{1}}, \ldots, \mathbf{e}_{\alpha_{d}}\right]^{\top} \in \mathbb{R}^{d \times M}$, then $\mathbf{E}_{[\boldsymbol{\alpha}]} \mathbf{A}$ selects the subset of rows in $\mathbf{A}$ indicated by $\boldsymbol{\alpha}$. For compactness, we use vec $\boldsymbol{\alpha}_{\boldsymbol{\alpha}}(\mathbf{A}) \triangleq$ $\operatorname{vec}\left(\mathbf{E}_{[\boldsymbol{\alpha}]} \mathbf{A}\right)$. The complementing subset of $\boldsymbol{\alpha}$ is indicated by $\boldsymbol{\alpha}^{c} \in \mathbb{N}^{M-d}$. A diagonal matrix with entries given by $\mathbf{d}$ is denoted by $\operatorname{Diag}(\mathbf{d})=\sum_{n=1}^{N} \mathbf{e}_{n} \mathbf{e}_{n}^{\top} \mathbf{d} \mathbf{e}_{n}^{\top}$. The square matrix, $\mathbf{A}$, has diagonal entries, $\operatorname{diag}(\mathbf{A})=\sum_{n=1}^{N} \mathbf{e}_{n} \mathbf{e}_{n}^{\top} \mathbf{A} \mathbf{e}_{n}$, a trace, $\operatorname{tr}(\mathbf{A})=\sum_{n=1}^{N}[\operatorname{diag}(\mathbf{A})]_{n}$, and a determinant, $\operatorname{det}(\mathbf{A})$. We indicate $\mathbf{A}-\mathbf{B}$ is positive definite using $\mathbf{A} \succ \mathbf{B}$ and positive semidefinite with $\mathbf{A} \succeq \mathbf{B}$. The operator $|\cdot|$ denotes the magnitude.

For a matrix $\mathbf{A}$ with block structure, the matrix $\mathbf{A}_{m, n}$ is the $m$ th row and $n$th column in the block representation of the matrix A using $M$ row partitions and $N$ column partitions. The special block diagonal matrix is necessarily a square matrix 
(implying $M=N$ ) that has off-diagonal partitions being zero, i.e., $\mathbf{A}_{m, n}=\mathbf{0}$ for $1 \leq m \neq n \leq M$, and is denoted with the direct sum notation, $\mathbf{A}=\mathbf{A}_{1,1} \oplus \mathbf{A}_{2,2} \oplus \ldots \oplus \mathbf{A}_{M, M}=\oplus \sum_{m=1}^{M} \mathbf{A}_{m, m}$, [25].

The common functions of random variables such as the expectation operator, entropy, and mutual information are denoted using $E\{\cdot\}, \mathcal{H}\{\cdot\}$, and $\mathcal{I}\{\cdot\}$, respectively. A random vector $\mathbf{x}$ following the normal distribution with mean $\boldsymbol{\mu}$ and covariance matrix $\boldsymbol{\Sigma}$ is denoted $\mathbf{x} \sim \mathcal{N}(\boldsymbol{\mu}, \boldsymbol{\Sigma})$. We use $\mathbf{x} \Perp \mathbf{y}$ to denote that a random vector $\mathbf{x}$ is independent of $\mathbf{y}$. We use standard elementary functions such as $\log (\cdot) \exp (\cdot), \Gamma(\cdot)$ for the natural logarithm, the anti-logarithm, and the complete Gamma function.

\section{IVA PROBLEM FormuLATION}

We begin by formulating the particular JBSS framework of interest, namely IVA in a more general manner than previously done [8], [9], [13], [13], [20], [24], [26]. The generalization allows analysis of IVA] when the samples are not iid, or alternatively when sample dependence is taken into account.

There are $K$ datasets, each containing $V$ samples, formed from the linear mixture of $N$ independent sources,

$$
\mathbf{X}^{[k]}=\mathbf{A}^{[k]} \mathbf{S}^{[k]} \in \mathbb{R}^{N \times V}, 1 \leq k \leq K .
$$

The entry in $n$th row and $v$ th column of $\mathbf{S}^{[k]}$ is $s_{n}^{[k]}(v)$, the $n$th row of $\mathbf{S}^{[k]}$ is denoted with the column vector $\mathbf{s}_{n}^{[k]}=$ $\left[s_{n}^{[k]}(1), \ldots, s_{n}^{[k]}(V)\right]^{\top} \in \mathbb{R}^{V}$, and the $v$ th column of $\mathbf{S}^{[k]}$ is denoted by the column vector $\mathbf{s}^{[k]}(v)=\left[s_{1}^{[k]}(v), \ldots, s_{N}^{[k]}(v)\right]^{\top} \in$ $\mathbb{R}^{N}$. The source matrices in each dataset can be concatenated to form $\mathbf{S}=\left[\left(\mathbf{S}^{[1]}\right)^{\top}, \ldots,\left(\mathbf{S}^{[K]}\right)^{\top}\right]^{\top} \in \mathbb{R}^{N K \times V}$. Using this notation, we can denote the JBSS data model with a single equation, namely $\mathbf{X}=\mathbf{A S}$, where $\mathbf{A}=\oplus \sum_{k=1}^{K} \mathbf{A}^{[k]}$. The invertible mixing matrices, $\mathbf{A}^{[k]} \in \mathbb{R}^{N \times N}$, and the sources $\mathbf{S}$ are unknown real-valued quantities to be estimated. The $n$th source component matrix (SCM) $\mathbf{S}_{n}=\left[\mathbf{s}_{n}^{[1]}, \ldots, \mathbf{s}_{n}^{[K]}\right]^{\top} \in \mathbb{R}^{K \times V}$, is independent of all other SCMs Then the probability distribution function (pdf) of the concatenated source vector, $\mathbf{S}$, can be written as $p(\mathbf{S})=\prod_{n=1}^{N} p_{n}\left(\mathbf{S}_{n}\right)$.

The IVA solution finds $K$ demixing matrices and the corresponding source estimates for each dataset, with the $k$ th ones denoted as $\mathbf{W}^{[k]}$ and $\mathbf{Y}^{[k]} \triangleq \mathbf{W}^{[k]} \mathbf{X}^{[k]}$, respectively. The estimate of the $n$th component from the $v$ th sample of the $k$ th dataset is given by $y_{n}^{[k]}(v)=\left(\mathbf{w}_{n}^{[k]}\right)^{\top} \mathbf{x}^{[k]}(v)=\sum_{l=1}^{N} w_{n, l}^{[k]} x_{l}^{[k]}(v)$, where $\left(\mathbf{w}_{n}^{[k]}\right)^{\top}$ is the $n$th row of $\mathbf{W}^{[k]}$. Furthermore, it is assumed that the mixing matrices possess no known relationship.

\section{IVA OBJECTIVE FUNCTION}

Just as in ICA the IVA objective function can be specified to be the maximization of the natural logarithm of the likelihood. Since $\mathbf{A}$ is block diagonal, the estimate of the $\hat{\mathbf{A}}^{-1}=\mathbf{W}=\oplus \sum_{k=1}^{K} \mathbf{W}^{[k]}$ is block diagonal and thus we choose in the sequel to use $\mathcal{W} \in \mathbb{R}^{N \times N \times K}$, i.e., a three-dimensional 'matrix', to denote the set of parameters to be estimated. We then have that

$$
\begin{aligned}
\mathcal{L}(\mathcal{W}) & \triangleq \log \left(p_{\mathbf{X}}(\mathbf{X})\right) \\
& =\log \left(\prod_{n=1}^{N} p_{n}\left(\mathbf{Y}_{n}\right)|\operatorname{det} \mathbf{W}|^{V}\right) \\
& =\sum_{n=1}^{N} \log \left(p_{n}\left(\mathbf{Y}_{n}\right)\right)+V \sum_{k=1}^{K} \log \left|\operatorname{det} \mathbf{W}^{[k]}\right|,
\end{aligned}
$$

where $p_{n}(\cdot)$ is the model for the distribution characterizing the multivariate source $\mathbf{S}_{n}$. Note that if $\mathbf{X}=\mathbf{A S}$, then vec $(\mathbf{S})=$ $\left(\mathbf{I}_{V} \otimes \mathbf{A}^{-1}\right) \operatorname{vec}(\mathbf{X})$, which implies $p_{\mathbf{X}}(\mathbf{X} ; \mathbf{A})=\left|\operatorname{det}\left(\mathbf{I}_{V} \otimes \mathbf{A}^{-1}\right)\right| p_{\mathbf{S}}\left(\left(\mathbf{I}_{V} \otimes \mathbf{A}^{-1}\right) \operatorname{vec}(\mathbf{X})\right)=\left|\operatorname{det} \mathbf{A}^{-1}\right|^{V} p_{\mathbf{S}}(\mathbf{S})$.

If we consider the case when $V \rightarrow \infty$, then we can define the source component vector (SCV) $\mathbf{s}_{n}$ as a random vector process and recall the definition of entropy rate [27, Eq 4.10] so that

$$
\mathcal{H}_{r}\left\{\mathbf{s}_{n}\right\} \triangleq \lim _{V \rightarrow \infty} \frac{1}{V} \mathcal{H}\left\{\mathbf{s}_{n}(1), \ldots, \mathbf{s}_{n}(V)\right\}=-\lim _{V \rightarrow \infty} \frac{1}{V} E\left\{\log p_{n}\left(\mathbf{S}_{n}\right)\right\} .
$$

By normalizing the likelihood objective function by $V$ and considering the limit,

$$
\begin{aligned}
\mathcal{C}_{\mathrm{IVA}}(\mathcal{W}) & \triangleq-\lim _{V \rightarrow \infty} \frac{1}{V} \mathcal{L}(\mathcal{W}) \\
& =\sum_{n=1}^{N} \mathcal{H}_{r}\left\{\mathbf{y}_{n}\right\}-\sum_{k=1}^{K} \log \left|\operatorname{det} \mathbf{W}^{[k]}\right| \\
& =\sum_{n=1}^{N}\left(\sum_{k=1}^{K} \mathcal{H}_{r}\left\{y_{n}^{[k]}\right\}-\mathcal{I}_{r}\left\{\mathbf{y}_{n}\right\}\right)-\sum_{k=1}^{K} \log \left|\operatorname{det} \mathbf{W}^{[k]}\right| .
\end{aligned}
$$


we can observe that IVA minimizes the entropy rate of the estimated SCVs (subject to the regularization term). This representation explains that the IVA objective function will equally weight the minimization of the source entropy rates and the maximization of the across dataset dependence measure provided by the mutual information rate of $\mathbf{y}_{n}$. It is also clear that the mutual information rate portion of the IVA objective function is responsible for resolving the permutation ambiguity across multiple datasets, since without the mutual information rate of the SCVs the objective function would be identical to using ICA on each of the $K$ datasets. This representation will be useful in our identifiability discussion in Section VII

In the sequel, we will use the multivariate score function $\boldsymbol{\Phi}_{n} \triangleq \boldsymbol{\Phi}_{n}\left(\mathbf{Y}_{n}\right)=-\partial \log \left(p_{n}\left(\mathbf{Y}_{n}\right)\right) / \partial \mathbf{Y}_{n} \in \mathbb{R}^{K \times V}$ and $\boldsymbol{\phi}_{n}^{[k]}=$ $\Phi_{n}^{\top} \mathbf{e}_{k}$.

\section{IVA FISHER INFORMATION MATRIX}

Here we derive the FIM of (1) with respect to (wrt) $\mathcal{W}$. The $K N^{2}$ parameters result in $K N^{2} \times K N^{2}$ dimension FIM with the entry associated with $w_{m_{1}, n_{1}}^{\left[k_{1}\right]}$ and $w_{m_{2}, n_{2}}^{\left[k_{2}\right]}$ denoted by and computed as:

$$
[\mathbf{F}(\mathcal{W})]_{k_{2}, m_{2}, n_{2}}^{k_{1}, m_{1}, n_{1}} \triangleq E\left\{\frac{\partial \mathcal{L}(\mathcal{W})}{\partial w_{m_{1}, n_{1}}^{\left[k_{1}\right]}} \frac{\partial \mathcal{L}(\mathcal{W})}{\partial w_{m_{2}, n_{2}}^{\left[k_{2}\right]}}\right\} .
$$

For the purposes of determining identifiability and the performance bound, we need only consider the FIM locally around a solution, i.e., $\mathbf{W}=\mathbf{A}^{-1}$, where $\mathbf{A}^{-1}$ and $\mathbf{W}$ are "freely" chosen as to alleviate all scale and permutation ambiguities. In general, this leads to a complex expression that depends on $\mathbf{A}$; fortunately this complexity is unnecessary. Due to the invariance of the induced Cramér-Rao lower bound (iCRLB) on $\mathbf{G}=\mathbf{W A}$ wrt the mixing matrix $\mathbf{A}=\oplus \sum_{k=1}^{K} \mathbf{A}^{[k]}$, we need only consider $\mathbf{A}=\mathbf{I}$, i.e., the Cramér-Rao lower bound (CRLB)] of $\mathbf{G}$ depends only on the statistics of the sources, [28]. Thus the matrix of interest is

$$
\left.[\mathbf{F}]_{k_{2}, m_{2}, n_{2}}^{k_{1}, m_{1}, n_{1}} \triangleq[\mathbf{F}(\mathcal{W})]_{k_{2}, m_{2}, n_{2}}^{k_{1}, m_{1}, n_{1}}\right|_{\mathbf{A}=\mathbf{I}, \mathbf{W}=\mathbf{I}} .
$$

It will prove useful to define $\mathcal{K}_{m, n}^{\left[k_{1}, k_{2}\right]} \triangleq \frac{1}{V} E\left\{\left(\phi_{m}^{\left[k_{1}\right]}\right)^{\top} \mathbf{s}_{n}^{\left[k_{1}\right]}\left(\mathbf{s}_{n}^{\left[k_{2}\right]}\right)^{\top} \phi_{m}^{\left[k_{2}\right]}\right\}, 1 \leq m, n \leq N$, to describe the form of the block diagonal FIM compactly. In Appendix A we show that the first $N$ block entries of the FIM are given by $\mathbf{F}_{n} \triangleq \operatorname{cov}\left\{\operatorname{diag}\left(\mathbf{\Phi}_{n} \mathbf{S}_{n}^{-}-\mathbf{I}_{V}\right)\right\}=V\left(\mathcal{K}_{n, n}-V \mathbf{1}_{K \times K}\right) \in \mathbb{R}^{K \times K}$ and the remaining block entries are defined for $n>m$ as

$$
\mathbf{F}_{m, n} \triangleq \operatorname{cov}\left\{\left[\begin{array}{c}
\operatorname{diag}\left(\mathbf{\Phi}_{m} \mathbf{S}_{n}^{\boldsymbol{\top}}\right) \\
\operatorname{diag}\left(\mathbf{\Phi}_{n} \mathbf{S}_{m}^{\top}\right)
\end{array}\right]\right\}=V\left[\begin{array}{cc}
\mathcal{K}_{m, n} & \mathbf{I}_{K} \\
\mathbf{I}_{K} & \mathcal{K}_{n, m}
\end{array}\right],
$$

where the $\left(k_{1}, k_{2}\right)$ entry of $\mathcal{K}_{m, n} \in \mathbb{R}^{K \times K}$ is $V^{-1} \operatorname{tr}\left(\boldsymbol{\Gamma}_{m}^{\left[k_{2}, k_{1}\right]} \mathbf{R}_{n}^{\left[k_{1}, k_{2}\right]}\right)$ when $m \neq n, \mathbf{R}_{n}^{\left[k_{1}, k_{2}\right]} \triangleq E\left\{\mathbf{s}_{n}^{\left[k_{1}\right]}\left(\mathbf{s}_{n}^{\left[k_{2}\right]}\right)^{\top}\right\} \in \mathbb{R}^{V \times V}$, and $\Gamma_{n}^{\left[k_{1}, k_{2}\right]} \triangleq E\left\{\phi_{n}^{\left[k_{1}\right]}\left(\phi_{n}^{\left[k_{2}\right]}\right)^{\top}\right\} \in \mathbb{R}^{V \times V}$.

The form of the FIM is a multivariate extension of the single dataset forms given in [2], [29]-[31]. The [FIM has a form that is a block matrix version of the single dataset result, e.g., see Fig. 5 and compare to the similar form given in [32] for complex-valued ICA The $2 \times 2$ blocks with ones in the off-diagonal elements and pair-wise cross terms in the two diagonal elements of the ICA FIM are here replaced with $2 \times 2$ block matrices with identity matrices in the off-diagonal blocks and the cross terms in the two diagonal block matrices, i.e., $\mathbf{F}_{m, n}$.

\section{IVA IDENTIFICATION CONDITIONS}

The identification of sources in (real-valued) ICA is possible so long as no two sources are Gaussian with proportional covariance matrices [2. Chapter 4]. When sources are said to be identifiable for ICA this means that the sources can be recovered up to a scale factor and arbitrary ordering, i.e., the true mixing matrix $\mathbf{A}_{0}$ can be identified upto $\mathbf{A}_{0} \boldsymbol{\Lambda} \mathbf{P}$, where $\boldsymbol{\Lambda}$ is any nonsingular diagonal matrix and $\mathbf{P}$ is any permutation matrix.

Since the the model structure of IVA is a generalization of the model structure for ICA, we expect a generalization of the identification conditions for ICA. Intuitively, the identification conditions for IVA are related to the dependence of the sources across the datasets. More specifically, when sources possess dependence across datasets we expect that these estimated sources can be 'aligned' - this is the original motivation of [VA [20], [22]. However, if there are sources for which no alignment exhibits dependence, then under the ICA identification conditions sources can be separated but not necessarily aligned. That is, without dependence across datasets the estimated sources of IVA would be no different than using ICA on each dataset individually since there is no dependency to exploit. The identification conditions, which we present in this section, capture both cases, i.e., when there is or is not dependence between sources across datasets.

To discuss identifiability of IVA we need to provide a notation that allows us to indicate a particular subset of rows in an SCM For this section, we let $\boldsymbol{\alpha}=\left[\alpha_{1} \ldots \alpha_{d_{\alpha}}\right]^{\top} \in \mathbb{N}^{K_{\alpha}}$, where $0 \leq K_{\alpha} \leq K$. The complementing subset of $\boldsymbol{\alpha}$ in $\{1, \ldots, K\}$ is indicated by $\boldsymbol{\alpha}^{c} \in \mathbb{N}^{K-K_{\alpha}}$. The IVA identification conditions use the following definition: 
Definition 1 ( $\boldsymbol{\alpha}$-Gaussian). A source, $\mathbf{S} \in \mathbb{R}^{K \times V}$, has an $\boldsymbol{\alpha}$-Gaussian component when $\operatorname{vec}_{\boldsymbol{\alpha}}(\mathbf{S}) \Perp \operatorname{vec}_{\boldsymbol{\alpha}^{c}}(\mathbf{S})$, and $\operatorname{vec}_{\boldsymbol{\alpha}}(\mathbf{S}) \sim$ $\mathcal{N}\left(\mathbf{0}, \mathbf{R}_{\alpha}\right)$, where $\mathbf{R}_{\alpha}=E\left\{\operatorname{vec}_{\boldsymbol{\alpha}}(\mathbf{S}) \operatorname{vec}_{\boldsymbol{\alpha}}^{\top}(\mathbf{S})\right\} \in \mathbb{R}^{K_{\alpha} V \times K_{\alpha} V}$ is nonsingular.

The $\alpha$-Gaussian definition is used to identify that there exist a subset of rows in an SCM that is independent of the other rows in the same $\mathrm{SCM}$ and that the given subset follows a multivariate Gaussian distribution. The theorem stating the IVA identification conditions and its proof follow.

Theorem 1 IVA Nonidentifiability). The sources cannot be identified if and only if (iff) $\exists \boldsymbol{\alpha} \neq \emptyset$ and $\exists m \neq n$ such that $\mathbf{S}_{m}$ and $\mathbf{S}_{n}$ have $\boldsymbol{\alpha}$-Gaussian components for which $\mathbf{R}_{m, \alpha}=\left(\mathbf{I}_{V} \otimes \mathbf{D}\right) \mathbf{R}_{n, \alpha}\left(\mathbf{I}_{V} \otimes \mathbf{D}\right) \in \mathbb{R}^{K_{\alpha} V \times K_{\alpha} V}$, where $\mathbf{D} \in \mathbb{R}^{K_{\alpha} \times K_{\alpha}}$ is any full rank diagonal matrix.

Proof of IVA Nonidentifiability: Given the FIM (40), (41), (42), since $\mathbf{F}_{m, n}$ is a covariance matrix, it must be positive semidefinite and is singular iff $\exists(\mathbf{a}, \mathbf{b}) \neq(\mathbf{0}, \mathbf{0}): \mathbf{a} \operatorname{diag}\left(\mathbf{\Phi}_{m} \mathbf{S}_{n}^{\top}\right)-\mathbf{b}^{\top} \operatorname{diag}\left(\mathbf{\Phi}_{n} \mathbf{S}_{m}^{\top}\right)=0, \forall \mathbf{S}_{m} \in \Omega_{\mathbf{S}_{m}}, \mathbf{S}_{n} \in \Omega_{\mathbf{S}_{n}}$, where $\Omega_{\mathbf{X}}$ denotes the sample space of the random matrix $\mathbf{X}$.

It is convenient to rewrite the following:

$$
\operatorname{diag}\left(\boldsymbol{\Phi}_{m} \mathbf{S}_{n}^{\boldsymbol{\top}}\right)=\operatorname{diag}\left(\sum_{v=1}^{V} \boldsymbol{\phi}_{m}(v) \mathbf{s}_{n}^{\boldsymbol{\top}}(v)\right)=\sum_{v=1}^{V} \boldsymbol{\phi}_{m}(v) \circ \mathbf{s}_{n}(v),
$$

where $\mathbf{s}_{n}(v)$ and $\phi_{m}(v)$ denote the $v$ th columns of $\mathbf{S}_{n}$ and $\mathbf{\Phi}_{m}$, respectively.

Hence, the following statements are all equivalent conditional on $\exists(\mathbf{a}, \mathbf{b}) \neq(\mathbf{0}, \mathbf{0})$ :

$$
\begin{aligned}
& \mathbf{F}_{m, n} \text { is singular } \\
& \Leftrightarrow \quad 0=\mathbf{a}^{\top} \operatorname{diag}\left(\boldsymbol{\Phi}_{m} \mathbf{S}_{n}^{\boldsymbol{\top}}\right)-\mathbf{b}^{\top} \operatorname{diag}\left(\boldsymbol{\Phi}_{n} \mathbf{S}_{m}^{\top}\right) \\
& \Leftrightarrow \quad 0=\mathbf{a}^{\top} \sum_{v=1}^{V} \phi_{m}(v) \circ \mathbf{s}_{n}(v)-\mathbf{b}^{\top} \sum_{q=1}^{V} \phi_{n}(q) \circ \mathbf{s}_{m}(q) \\
& \Leftrightarrow \quad 0=\left(\mathbf{1}_{V} \otimes \mathbf{a}\right)^{\top}\left(\operatorname{vec}\left(\boldsymbol{\Phi}_{m}\right) \circ \operatorname{vec}\left(\mathbf{S}_{n}\right)\right)-\left(\mathbf{1}_{V} \otimes \mathbf{b}\right)^{\top}\left(\operatorname{vec}\left(\boldsymbol{\Phi}_{n}\right) \circ \operatorname{vec}\left(\mathbf{S}_{m}\right)\right) \\
& \Leftrightarrow \quad 0=\operatorname{vec}_{\boldsymbol{\alpha}}^{\top}\left(\boldsymbol{\Phi}_{m}\right)\left(\mathbf{I}_{V} \otimes \mathbf{D}_{\mathbf{a}[\boldsymbol{\alpha}]}\right) \operatorname{vec}_{\boldsymbol{\alpha}}\left(\mathbf{S}_{n}\right)-\operatorname{vec}_{\boldsymbol{\beta}}^{\top}\left(\boldsymbol{\Phi}_{n}\right)\left(\mathbf{I}_{V} \otimes \mathbf{D}_{\mathbf{b}[\boldsymbol{\beta}]}\right) \operatorname{vec}_{\boldsymbol{\beta}}\left(\mathbf{S}_{m}\right) \\
& \Leftrightarrow \quad 0=\operatorname{vec}_{\boldsymbol{\alpha}}^{\top}\left(\boldsymbol{\Phi}_{m}\right)\left(\mathbf{I}_{V} \otimes \mathbf{D}_{\mathbf{a}[\boldsymbol{\alpha}]}\right) \operatorname{vec}_{\boldsymbol{\alpha}}\left(\mathbf{S}_{n}\right)-\operatorname{vec}_{\boldsymbol{\alpha}}^{\top}\left(\boldsymbol{\Phi}_{n}\right)\left(\mathbf{I}_{V} \otimes \mathbf{D}_{\mathbf{b}[\boldsymbol{\alpha}]}\right) \operatorname{vec}_{\boldsymbol{\alpha}}\left(\mathbf{S}_{m}\right) \\
& \Leftrightarrow \quad 0=\operatorname{vec}_{\boldsymbol{\alpha}}^{\top}\left(\mathbf{S}_{m}\right) \mathbf{R}_{m, \alpha}^{-1}\left(\mathbf{I}_{V} \otimes \mathbf{D}_{\mathbf{a}[\boldsymbol{\alpha}]}\right) \operatorname{vec}_{\boldsymbol{\alpha}}\left(\mathbf{S}_{n}\right)-\operatorname{vec}_{\boldsymbol{\alpha}}^{\top}\left(\mathbf{S}_{n}\right) \mathbf{R}_{n, \boldsymbol{\alpha}}^{-1}\left(\mathbf{I}_{V} \otimes \mathbf{D}_{\mathbf{b}[\boldsymbol{\alpha}]}\right) \operatorname{vec}_{\boldsymbol{\alpha}}\left(\mathbf{S}_{m}\right) \\
& \Leftrightarrow \quad 0=\mathbf{R}_{m, \alpha}^{-1}\left(\mathbf{I}_{V} \otimes \mathbf{D}_{\mathbf{a}[\boldsymbol{\alpha}]}\right)-\left(\mathbf{I}_{V} \otimes \mathbf{D}_{\mathbf{b}[\boldsymbol{\alpha}]}\right) \mathbf{R}_{n, \alpha}^{-1} \\
& \Leftrightarrow \quad \mathbf{R}_{m, \alpha}=\left(\mathbf{I}_{V} \otimes \mathbf{D}_{\mathbf{a}[\boldsymbol{\alpha}]}\right) \mathbf{R}_{n, \alpha}\left(\mathbf{I}_{V} \otimes \mathbf{D}_{\mathbf{b}[\boldsymbol{\alpha}]}^{-1}\right) \\
& \Leftrightarrow \quad \mathbf{R}_{m, \alpha}=\left(\mathbf{I}_{V} \otimes \mathbf{D}\right) \mathbf{R}_{n, \alpha}\left(\mathbf{I}_{V} \otimes \mathbf{D}\right),
\end{aligned}
$$

where $\mathbf{D}_{\mathbf{a}[\boldsymbol{\alpha}]} \triangleq \operatorname{Diag}(\mathbf{a}[\boldsymbol{\alpha}]), \mathbf{D}_{\mathbf{b}[\boldsymbol{\alpha}]} \triangleq \operatorname{Diag}(\mathbf{b}[\boldsymbol{\alpha}]), \boldsymbol{\alpha} \in \mathbb{N}^{K_{\alpha}}$, and $\boldsymbol{\beta} \in \mathbb{N}^{K_{\beta}}$.

It is straightforward to observe that (8), (9), (10), and (11) are equivalent expressions. From the relationship $(\mathbf{x} \otimes \mathbf{y})^{\top}(\mathbf{w} \circ \mathbf{z})=$ $\mathbf{w}^{\top}(\operatorname{Diag}(\mathbf{x}) \otimes \operatorname{Diag}(\mathbf{y})) \mathbf{z}$, the expression in (12) holds only when $\boldsymbol{\alpha}=\boldsymbol{\beta}$, i.e., the zero entries of $\mathbf{a}$ and $\mathbf{b}$ are at the same locations. See Lemma 1 below to explain (14). Since (14) must hold for all possible values of $\operatorname{vec}_{\boldsymbol{\alpha}}\left(\mathbf{S}_{m}\right)$ and $\operatorname{vec}_{\boldsymbol{\alpha}}\left(\mathbf{S}_{n}\right)$, 15. must hold. Equation (16) is equivalent since all entries of $\mathbf{b}[\boldsymbol{\alpha}]$ are nonzero by [13). Lastly, since $\mathbf{R}_{m, \alpha}$ is symmetric we must have that either $\mathbf{R}_{n, \alpha}$ is diagonal or $\mathbf{D}_{\mathbf{a}[\boldsymbol{\alpha}]}=\left(\mathbf{D}_{\mathbf{b}[\boldsymbol{\alpha}]}\right)^{-1}$. In either case 17] holds.

Lemma 1. For $m \neq n$,

$$
\operatorname{vec}_{\boldsymbol{\alpha}}^{\top}\left(\boldsymbol{\Phi}_{m}\right)\left(\mathbf{I}_{V} \otimes \mathbf{D}_{\mathbf{a}[\boldsymbol{\alpha}]}\right) \operatorname{vec}_{\boldsymbol{\alpha}}\left(\mathbf{S}_{n}\right)=\operatorname{vec}_{\boldsymbol{\alpha}}^{\top}\left(\boldsymbol{\Phi}_{n}\right)\left(\mathbf{I}_{V} \otimes \mathbf{D}_{\mathbf{b}[\boldsymbol{\alpha}]}\right) \operatorname{vec}_{\boldsymbol{\alpha}}\left(\mathbf{S}_{m}\right)
$$

holds iff

$$
\operatorname{vec}_{\boldsymbol{\alpha}}^{\top}\left(\mathbf{S}_{m}\right) \mathbf{R}_{m, \alpha}^{-1}\left(\mathbf{I}_{V} \otimes \mathbf{D}_{\mathbf{a}[\boldsymbol{\alpha}]}\right) \operatorname{vec}_{\boldsymbol{\alpha}}\left(\mathbf{S}_{n}\right)=\operatorname{vec}_{\boldsymbol{\alpha}}^{\top}\left(\mathbf{S}_{n}\right) \mathbf{R}_{n, \alpha}^{-1}\left(\mathbf{I}_{V} \otimes \mathbf{D}_{\mathbf{b}[\boldsymbol{\alpha}]}\right) \operatorname{vec}_{\boldsymbol{\alpha}}\left(\mathbf{S}_{m}\right)
$$

and $\mathbf{S}_{m}$ and $\mathbf{S}_{n}$ each have an $\boldsymbol{\alpha}$-Gaussian component.

Proof: $(\Rightarrow)$ Since the left-hand side of $[18)$ is linear in $\operatorname{vec}_{\boldsymbol{\alpha}}\left(\mathbf{S}_{n}\right)$ we must have that $\operatorname{vec}_{\boldsymbol{\alpha}}\left(\boldsymbol{\Phi}_{n}\right)$ is not a function of $\operatorname{vec}_{\boldsymbol{\alpha}^{c}}\left(\mathbf{S}_{n}\right)$ and it is necessarily linear in $\operatorname{vec}_{\boldsymbol{\alpha}}\left(\mathbf{S}_{n}\right)$, i.e., $\mathbf{S}_{n}$ has $\boldsymbol{\alpha}$-Gaussian component. By symmetry, the same can be concluded about $\mathbf{S}_{m}$.

$(\Leftarrow)$ If $\mathbf{S}_{n}$ has $\boldsymbol{\alpha}$-Gaussian component then $\operatorname{vec}_{\boldsymbol{\alpha}}\left(\mathbf{\Phi}_{n}\right)=\mathbf{R}_{n}^{-1} \mathrm{vec}_{\boldsymbol{\alpha}}\left(\mathbf{S}_{n}\right)$.

It is noteworthy to mention that the IVA identification conditions admit sources for which the distribution can be factored, i.e., $p_{n}\left(\mathbf{S}_{n}\right)=\prod_{q=1}^{Q} p_{n_{q}}\left(\operatorname{vec}_{\mathcal{Q}_{q}}\left(\mathbf{S}_{n}\right)\right)$, where $\left\{\mathcal{Q}_{1}, \mathcal{Q}_{2}, \ldots, \mathcal{Q}_{Q}\right\}, \mathcal{Q}_{q} \subset\{1, \ldots, K\}, \mathcal{Q}_{q} \cap \mathcal{Q}_{q^{\prime}}=\emptyset \forall q \neq q^{\prime}$, and $\cup_{q=1}^{Q} \mathcal{Q}_{q}=$ $\{1, \ldots, K\}$. If, for example $Q=K$, then IVA would produce the same identification conditions as ICA on each dataset individually. Stated differently, identifiability of IVA does not require the sources to possess dependence across datasets. 
Recalling that a prime motivation for considering the IVA formulation is to determine when the sources can be aligned in a common way across all datasets, i.e., under what conditions is $\hat{\mathbf{A}}^{[k]}=\left(\mathbf{W}^{[k]}\right)^{-1}=\mathbf{A}[k] \mathbf{P} \boldsymbol{\Lambda}^{[k]}$, where $\boldsymbol{\Lambda}^{[k]}$ is any full rank diagonal matrix and $\mathbf{P}$ is a permutation matrix commonly shared by all datasets. The common permutation identification condition is given in the next theorem which uses the following definition:

Definition 2 ( $\boldsymbol{\alpha}$-independent). A source, $\mathbf{S} \in \mathbb{R}^{K \times V}$, is $\boldsymbol{\alpha}$-independent when $\operatorname{vec}_{\boldsymbol{\alpha}}(\mathbf{S}) \Perp \operatorname{vec}_{\boldsymbol{\alpha}^{c}}(\mathbf{S})$.

The $\alpha$-independent definition is used to identify that there exist a subset of rows in an SCM (or SCV) that is independent of the other rows in the SCM SCV.

Theorem 2 (Common Permutation Matrix for IVA). Assuming the IVA identification conditions of Theorem 1 are satisfied, i.e., in the limit as $V \rightarrow \infty$ so that $\left(\mathbf{W}^{[k]}\right)^{-1}=\mathbf{A}^{[k]} \mathbf{P}^{[k]} \Lambda^{[k]}$ :

The permutation matrix associated with each dataset is common iff $\forall m \neq n \nexists \boldsymbol{\alpha} \neq \emptyset$ such that both $\mathbf{s}_{m}$ and $\mathbf{s}_{n}$ are $\boldsymbol{\alpha}$-independent.

Proof: The objective function given in (3) makes it clear that any permutation matrix at most effects the $\mathcal{I}_{r}\left\{\mathbf{y}_{n}\right\}$ term. Furthermore, we only need consider permutation matrices that can achieve the global minimum. The proof is by contradiction (in both directions):

$$
\begin{array}{rrr} 
& \exists 1 \leq k_{1} \neq k_{2} \leq K: \mathbf{P}^{\left[k_{1}\right]} \neq \mathbf{P}^{\left[k_{2}\right]} \\
\Leftrightarrow & \mathcal{I}_{r}\left\{\mathbf{s}_{m}^{[\boldsymbol{\alpha}]} ; \mathbf{s}_{n}^{\left[\boldsymbol{\alpha}^{c}\right]}\right\}+\mathcal{I}_{r}\left\{\mathbf{s}_{n}^{[\boldsymbol{\alpha}]} ; \mathbf{s}_{m}^{\left[\boldsymbol{\alpha}^{c}\right]}\right\} & =\mathcal{I}_{r}\left\{\mathbf{s}_{m}^{[\boldsymbol{\alpha}]} ; \mathbf{s}_{m}^{\left[\boldsymbol{\alpha}^{c}\right]}\right\}+\mathcal{I}_{r}\left\{\mathbf{s}_{n}^{[\boldsymbol{\alpha}]} ; \mathbf{s}_{n}^{\left[\boldsymbol{\alpha}^{c}\right]}\right\} \\
\Leftrightarrow & 0=\mathcal{I}_{r}\left\{\mathbf{s}_{m}^{[\boldsymbol{\alpha}]} ; \mathbf{s}_{m}^{\left[\boldsymbol{\alpha}^{c}\right]}\right\}+\mathcal{I}_{r}\left\{\mathbf{s}_{n}^{[\boldsymbol{\alpha}]} ; \mathbf{s}_{n}^{\left[\boldsymbol{\alpha}^{c}\right]}\right\} \\
\Leftrightarrow & \mathbf{S}_{m} \text { and } \mathbf{S}_{n} \text { are } \boldsymbol{\alpha} \text {-independent }
\end{array}
$$

We have used the fact that $\mathcal{I}_{r}\{\mathcal{X} ; \mathcal{Y}\} \geq 0$ with equality iff $\mathcal{X} \Perp \mathcal{Y}$, which implies by the assumption of IVA that $\mathcal{I}_{r}\left\{\mathbf{s}_{i}^{\left[\boldsymbol{\alpha}_{1}\right]} ; \mathbf{s}_{j}^{\left[\boldsymbol{\alpha}_{2}\right]}\right\}=0 \forall i \neq j, \boldsymbol{\alpha}_{1}, \boldsymbol{\alpha}_{2}$, where $\boldsymbol{\alpha}_{1}$ and $\boldsymbol{\alpha}_{2}$ are any indexing sets.

Thus, Theorem 2 provides an additional restriction on the sources (in a pairwise manner) which is required when the estimated dependent sources across all datasets are to be 'aligned'.

\section{A. Special Cases}

It is now insightful to consider important special cases of IVA with regard to the identification conditions. We begin by considering the case when the $V$ samples are iid This is equivalent to having $V=1$, which implies that the identification conditions can be derived as a special case of Theorem 1 .

Theorem 3 IVA Nonidentifiability with iid Samples). The sources cannot be identified iff $\exists \boldsymbol{\alpha} \neq \emptyset$ and $\exists m \neq n$ such that $\mathbf{s}_{m}$ and $\mathbf{s}_{n}$ have $\boldsymbol{\alpha}$-Gaussian components and $\mathbf{R}_{m, \alpha}=\mathbf{D} \mathbf{R}_{n, \alpha} \mathbf{D} \in \mathbb{R}^{K_{\alpha} \times K_{\alpha}}$, where $\mathbf{D}$ is any full rank diagonal matrix.

Another special case of interest is when $K=1$, yielding the same formulation as ICA assuming sample-to-sample dependence, i.e., not iid samples, the most general form for real-valued ICA

Theorem 4 (ICA Nonidentifiability [2], [33]). The sources cannot be identified iff $\exists m \neq n$ such that $\mathbf{s}_{m} \in \mathbb{R}^{V}$ and $\mathbf{s}_{n} \in \mathbb{R}^{V}$ are Gaussian and $\mathbf{R}_{m}=\delta^{2} \mathbf{R}_{n} \in \mathbb{R}^{V \times V}$, where $\delta \neq 0$.

It can be verified that the identification conditions of Theorem 4 are consistent with the results found in [2, Chapter 4] and [33].

Another special case of interest is when $K=1$, and assuming iid samples.

Theorem 5 (ICA Nonidentifiability with iid Samples [34]). The sources cannot be identified iff] $\exists m \neq n$ such that $s_{m} \in \mathbb{R}$ and $s_{n} \in \mathbb{R}$ are Gaussian.

The claim of Theorem 5, originally given in [34], states the well known result for ICA that at most one source can be Gaussian for identification of all iid sources. Algorithms based on the iid assumption using higher-order statistics have been the most widely exploited type of diversity in the derivation of ICA algorithms.

Additional diversity can extend the IVA and ICA identification conditions. An example is when data is complex-valued, a case we do not consider in this paper.

\section{CRLB AND ICRLB}

The CRLB associated with the parameter vector $\boldsymbol{\Theta}$ is the inverse of the FIM i.e., cov $\{\hat{\boldsymbol{\Theta}}\} \geq \mathbf{F}^{-1}$, where $\hat{\boldsymbol{\Theta}}$ is an estimator for $\Theta$. Due to the block diagonal structure of 40 ) we have that the inverse (if it exists, see identifiability discussion in Section 
VII of the portion of the FIM associated with the $m$ th and $n$th source denoted by $\mathbf{F}_{m, n}$ in 6 is

$$
\mathbf{F}_{m, n}^{-1}=\frac{1}{V}\left[\begin{array}{cc}
\left(\mathcal{K}_{m, n}-\mathcal{K}_{n, m}^{-1}\right)^{-1} & * \\
* & \left(\mathcal{K}_{n, m}-\mathcal{K}_{m, n}^{-1}\right)^{-1}
\end{array}\right] .
$$

It yields the following $\overline{C R L B}$ on the estimates of the demixing matrix quantities,

$$
\operatorname{var}\left\{w_{m, n}^{[k]}\right\} \geq \frac{1}{V} \mathbf{e}_{k}^{\top}\left(\mathcal{K}_{m, n}-\mathcal{K}_{n, m}^{-1}\right)^{-1} \mathbf{e}_{k}, 1 \leq m \neq n \leq N
$$

For this JBSS formulation, the definition of the interference to source ratio (ISR) is the same as in BSS [2], [31], namely:

$$
\operatorname{ISR}_{m, n}^{[k]} \triangleq E\left\{\left(g_{m, n}^{[k]}\right)^{2}\right\} \frac{E\left\{\left|\mathbf{s}_{n}^{[k]}\right|^{2}\right\}}{E\left\{\left|\mathbf{s}_{m}^{[k]}\right|^{2}\right\}}, 1 \leq m \neq n \leq N,
$$

where $g_{m, n}^{[k]}=\mathbf{e}_{m}^{\top} \mathbf{G}^{[k]} \mathbf{e}_{n}$ and $\mathbf{G}^{[k]} \triangleq \mathbf{W}^{[k]} \mathbf{A}^{[k]}$ is called the $k$ th global demixing-mixing matrix.

The ICRLB for ISR is then:

$$
\operatorname{ISR}_{m, n}^{[k]} \geq \frac{1}{V} \mathbf{e}_{k}^{\top}\left(\mathcal{K}_{m, n}-\mathcal{K}_{n, m}^{-1}\right)^{-1} \mathbf{e}_{k} \frac{E\left\{\left|\mathbf{s}_{n}^{[k]}\right|^{2}\right\}}{E\left\{\left|\mathbf{s}_{m}^{[k]}\right|^{2}\right\}} .
$$

Since the sources are (potentially) multivariate in the IVA formulation, it makes sense to define the ISR according to

$$
\mathrm{ISR}_{m, n} \triangleq \sum_{k=1}^{K} \operatorname{ISR}_{m, n}^{[k]}, 1 \leq m \neq n \leq N
$$

After some simple manipulation, the following compact form for the iCRLB results:

$$
\mathrm{ISR}_{m, n} \geq \frac{1}{V} \operatorname{tr}\left(\left(\mathcal{K}_{m, n}-\mathcal{K}_{n, m}^{-1}\right)^{-1} \circ \mathbf{C}_{n} \oslash \mathbf{C}_{m}\right),
$$

where $\mathbf{C}_{n} \triangleq E\left\{\mathbf{S}_{n} \mathbf{S}_{n}^{\top}\right\} \in \mathbb{R}^{K \times K}$. In what follows, for notational simplicity and without loss of generality, we assume the sources have equal energy within each dataset, i.e., $\operatorname{diag}\left(\mathbf{C}_{n}\right)=\operatorname{diag}\left(\mathbf{C}_{m}\right) \forall 1 \leq m, n \leq N$.

When the samples are iid, then the IVA iCRLB simplifies further if we note that:

$$
\begin{gathered}
\mathbf{R}_{n}^{\left[k_{1}, k_{2}\right]}=E\left\{\mathbf{s}_{n}^{\left[k_{1}\right]}\left(\mathbf{s}_{n}^{\left[k_{2}\right]}\right)^{\top}\right\}=\sigma_{n}^{\left[k_{1}, k_{2}\right]} \mathbf{I}_{V}, \\
\boldsymbol{\Gamma}_{m}^{\left[k_{1}, k_{2}\right]}=E\left\{\boldsymbol{\phi}_{m}^{\left[k_{1}\right]}\left(\boldsymbol{\phi}_{m}^{\left[k_{2}\right]}\right)^{\top}\right\}=\gamma_{m}^{\left[k_{1}, k_{2}\right]} \mathbf{I}_{V},
\end{gathered}
$$

and for $1 \leq m \neq n \leq N$,

$$
\mathcal{K}_{m, n}^{\left[k_{1}, k_{2}\right]}=\frac{1}{V} \operatorname{tr}\left(\boldsymbol{\Gamma}_{m}^{\left[k_{2}, k_{1}\right]} \mathbf{R}_{n}^{\left[k_{1}, k_{2}\right]}\right)=\gamma_{m}^{\left[k_{1}, k_{2}\right]} \sigma_{n}^{\left[k_{1}, k_{2}\right]},
$$

where $\sigma_{n}^{\left[k_{1}, k_{2}\right]} \triangleq E\left\{s_{n}^{\left[k_{1}\right]}(v) s_{n}^{\left[k_{2}\right]}(v)\right\} \in \mathbb{R}$ and $\gamma_{m}^{\left[k_{1}, k_{2}\right]} \triangleq E\left\{\phi_{m}^{\left[k_{1}\right]}(v) \phi_{m}^{\left[k_{2}\right]}(v)\right\} \in \mathbb{R}$ are not dependent on $v$ due to the iid assumption.

For the lid IVA discussion we simplify by replacing the $\mathrm{SCM}$ notation with $\mathrm{SCV}$ notation, i.e., we define the $\mathrm{SCV}$, $\mathbf{s}_{n}$, as a random vector with $V$ realizations denoted by $\mathbf{s}_{n}(v) \in \mathbb{R}^{K}$. In addition, the multivariate score function is denoted by $\phi_{m}\left(\mathbf{s}_{m}\right) \in \mathbb{R}^{K}$. For now, let $\mathbf{R}_{n}=E\left\{\mathbf{s}_{n} \mathbf{s}_{n}^{\top}\right\} \in \mathbb{R}^{K \times K}$ and $\boldsymbol{\Gamma}_{m}=E\left\{\phi_{m}\left(\mathbf{s}_{m}\right) \phi_{m}^{\top}\left(\mathbf{s}_{m}\right)\right\} \in \mathbb{R}^{K \times K}$, from which we observe that $\mathcal{K}_{m, n}=\boldsymbol{\Gamma}_{m} \circ \mathbf{R}_{n}=\operatorname{var}\left\{\phi_{m}\left(\mathbf{s}_{m}\right) \circ \mathbf{s}_{n}\right\}$.

The above gives the following $\mathrm{iCRLB}$ on the estimates of the demixing matrix entries when the samples are iid,

$$
\operatorname{ISR}_{m, n} \geq \frac{1}{V} \operatorname{tr}\left(\left(\boldsymbol{\Gamma}_{m} \circ \mathbf{R}_{n}-\left(\boldsymbol{\Gamma}_{n} \circ \mathbf{R}_{m}\right)^{-1}\right)^{-1}\right) .
$$

The relationship between $\boldsymbol{\Gamma}$ and $\mathbf{R}$ given in the following lemma is the multivariate extension of the result given by [30 Lemma $1 \mathrm{~b}$ of Appendix B], which has also been given in [2, Chapter 4].

Lemma 2. $\boldsymbol{\Gamma} \succeq \mathbf{R}^{-1}$, with equality $\left[\right.$ ff] $\phi=\mathbf{R}^{-1} \mathbf{s}$, i.e., $\mathbf{s}$ follows the Gaussian distribution. 
Proof: The proof applies the extension of the Cauchy-Schwarz inequality for covariance matrices as given in [35]. Specifically, $\boldsymbol{\Gamma}-E\left\{\boldsymbol{\phi} \mathbf{s}^{\top}\right\} \mathbf{R}^{-1} E\left\{\mathbf{s} \phi^{\top}\right\} \succeq \mathbf{0}$, with equality iff $\boldsymbol{\phi}=E\left\{\boldsymbol{\phi} \mathbf{s}^{\top}\right\} \mathbf{R}^{-1} \mathbf{s}$. By noting that $E\left\{\mathbf{s} \boldsymbol{T}^{\top}\right\}=\mathbf{I}$ we arrive at the assertion.

From this lemma, we see that a measure of non-Gaussianity (or higher-order statistics) is captured by the 'difference' between $\boldsymbol{\Gamma}$ and $\mathbf{R}^{-1}$. Next, we show for elliptical distributions - a broad class of source distributions-how this non-Gaussianity measure can be captured by a scalar quantity.

The pdf (assuming it exists) for a zero-mean random vector following the elliptical distribution is

$$
p(\mathbf{x})=\frac{c_{K}}{\sqrt{\operatorname{det} \mathbf{\Sigma}}} h_{e}\left(\mathbf{x}^{\top} \mathbf{\Sigma}^{-1} \mathbf{x}\right)
$$

where $\boldsymbol{\Sigma} \in \mathbb{R}^{K \times K}$ is the positive definite matrix frequently termed the dispersion matrix, $h_{e}$ is some nonnegative function, and $c_{K}$ denotes the constant that makes (29) integrate to one. If the covariance matrix, $E\left\{\mathbf{x} \mathbf{x}^{\top}\right\}=\mathbf{R}$, exists, then for any elliptical distribution it is a scalar multiple of the dispersion matrix, i.e., $\mathbf{R}=\rho \boldsymbol{\Sigma}$, where $\rho>0$. Then the score function, $\boldsymbol{\phi}(\mathbf{x}) \triangleq-\partial \log p(\mathbf{x}) / \partial \mathbf{x}=g\left(\mathbf{x}^{\top} \boldsymbol{\Sigma}^{-1} \mathbf{x}\right) \boldsymbol{\Sigma}^{-1} \mathbf{x}$, where $g(u)=-2 \frac{1}{h_{e}(u)} \frac{d h_{e}(u)}{d u}$.

For elliptical distributions (see Appendix B, $\boldsymbol{\Gamma}=\kappa \mathbf{R}^{-1}, K \geq 2$, where $\kappa \triangleq E\left\{g^{2}\left(r^{2}\right) r^{K+1}\right\} \frac{2 \pi^{K / 2}}{K \Gamma(K / 2)} \rho$. By application of Lemma 2 this implies that $\kappa \geq 1$ with equality iff Gaussian ${ }^{1}$ Therefore, the iCRLB for ISR with elliptical sources is

$$
\operatorname{ISR}_{m, n} \geq \frac{1}{V} \operatorname{tr}\left(\left(\kappa_{m} \mathbf{R}_{m}^{-1} \circ \mathbf{R}_{n}-\left(\kappa_{n} \mathbf{R}_{n}^{-1} \circ \mathbf{R}_{m}\right)^{-1}\right)^{-1}\right) .
$$

For this performance bound we provide the following theorem.

Theorem 6. If two SCVS follow distributions from the elliptical family with covariance matrices, $\mathbf{R}_{m}$ and $\mathbf{R}_{n}$, then ISR $R_{m, n}$ is less than or equal to the $I_{S R_{m, n}}$ associated with Gaussian $S C V s$ having the same covariance matrices.

Proof: See [8] for proof that $\mathbf{R}_{m}^{-1} \circ \mathbf{R}_{n}-\left(\mathbf{R}_{n}^{-1} \circ \mathbf{R}_{m}\right)^{-1} \succeq 0$. For elliptically distributed sources, via Lemma 2 , we have that $\kappa_{m} \geq 1$ and $\kappa_{n} \geq 1$, thus

$$
\begin{gathered}
\kappa_{m} \mathbf{R}_{m}^{-1} \circ \mathbf{R}_{n}-\kappa_{n}^{-1}\left(\mathbf{R}_{n}^{-1} \circ \mathbf{R}_{m}\right)^{-1} \succeq \mathbf{R}_{m}^{-1} \circ \mathbf{R}_{n}-\left(\mathbf{R}_{n}^{-1} \circ \mathbf{R}_{m}\right)^{-1} \\
\left(\kappa_{m} \mathbf{R}_{m}^{-1} \circ \mathbf{R}_{n}-\kappa_{n}^{-1}\left(\mathbf{R}_{n}^{-1} \circ \mathbf{R}_{m}\right)^{-1}\right)^{-1} \preceq\left(\mathbf{R}_{m}^{-1} \circ \mathbf{R}_{n}-\left(\mathbf{R}_{n}^{-1} \circ \mathbf{R}_{m}\right)^{-1}\right)^{-1}
\end{gathered}
$$

and since $\mathbf{A} \preceq \mathbf{B}$, it implies $\mathbf{x}^{\top} \mathbf{A} \mathbf{x} \leq \mathbf{x}^{\top} \mathbf{B} \mathbf{x}, \forall \mathbf{x}$, and thus $\operatorname{tr}(\mathbf{A}) \leq \operatorname{tr}(\mathbf{B})$.

A special case, which arrives at a form directly analogous to the ICA form, occurs when $\mathbf{R}_{m}=\mathbf{R}_{n}=\mathbf{I}_{K}$ :

$$
\operatorname{ISR}_{m, n} \geq \frac{K}{V} \frac{\kappa_{n}}{\kappa_{m} \kappa_{n}-1}
$$

This expression clearly shows how for second-order uncorrelated elliptical sources, the 'degree' of non-Gaussianity as expressed by $\kappa$, directly determines the source separation performance. In fact, as shown in the following theorem, the same statement holds for second-order correlated elliptical sources.

Theorem 7. If three SCVs follow distributions from the elliptical family with covariance matrices, $\mathbf{R}_{m}=\mathbf{R}_{m}$, and $\mathbf{R}_{n}$, and $\kappa_{m} \geq \kappa_{m^{\prime}}$ then $I S R_{m, n} \leq I S R_{m^{\prime}, n}$.

Proof: For elliptically distributed sources, via Lemma 2, we have that $\kappa_{m} \geq \kappa_{m^{\prime}} \geq 1$ and $\kappa_{n} \geq 1$, thus

$$
\begin{gathered}
\kappa_{m} \mathbf{R}_{m}^{-1} \circ \mathbf{R}_{n}-\kappa_{n}^{-1}\left(\mathbf{R}_{n}^{-1} \circ \mathbf{R}_{m}\right)^{-1} \succeq \kappa_{m^{\prime}} \mathbf{R}_{m}^{-1} \circ \mathbf{R}_{n}-\kappa_{n}^{-1}\left(\mathbf{R}_{n}^{-1} \circ \mathbf{R}_{m}\right)^{-1} \\
\left(\kappa_{m} \mathbf{R}_{m}^{-1} \circ \mathbf{R}_{n}-\kappa_{n}^{-1}\left(\mathbf{R}_{n}^{-1} \circ \mathbf{R}_{m}\right)^{-1}\right)^{-1} \preceq\left(\kappa_{m^{\prime}} \mathbf{R}_{m}^{-1} \circ \mathbf{R}_{n}-\kappa_{n}^{-1}\left(\mathbf{R}_{n}^{-1} \circ \mathbf{R}_{m}\right)^{-1}\right)^{-1}
\end{gathered}
$$

and since $\mathbf{A} \preceq \mathbf{B}$ implies $\mathbf{x}^{\top} \mathbf{A} \mathbf{x} \leq \mathbf{x}^{\top} \mathbf{B} \mathbf{x}, \forall \mathbf{x}$, and thus $\operatorname{tr}(\mathbf{A}) \leq \operatorname{tr}(\mathbf{B})$.

\section{A. CRLB for ICA}

Another special case, which is of particular interest, is when there is only one dataset, i.e., $K=1$. For this case, the expressions above further simplify to the more extensively studied [CA performance bounds [2], [29]-[31]. If $K=1$, we can replace the SCM notation with source component notation, i.e., let $\mathbf{s}_{n} \in \mathbb{R}^{V}$ be the random vector and the multivariate score function be denoted by $\phi_{m} \in \mathbb{R}^{V}$. Then, for this section we have $\mathbf{R}_{n}=E\left\{\mathbf{s}_{n} \mathbf{s}_{n}^{\top}\right\} \in \mathbb{R}^{V \times V}$ and $\boldsymbol{\Gamma}_{m}=E\left\{\boldsymbol{\phi}_{m} \boldsymbol{\phi}_{m}^{\top}\right\} \in \mathbb{R}^{V \times V}$, from which we observe that for $m \neq n, \mathcal{K}_{m, n}=\mathcal{K}_{m, n}=V^{-1} \operatorname{tr}\left(\boldsymbol{\Gamma}_{m} \mathbf{R}_{n}\right)=V^{-1} \operatorname{var}\left\{\boldsymbol{\phi}_{m}^{\top} \mathbf{s}_{n}\right\}$. Also,

$$
\mathbf{F}_{m, n}=V\left[\begin{array}{cc}
\frac{1}{V} \operatorname{var}\left\{\boldsymbol{\phi}_{m}^{\top} \mathbf{s}_{n}\right\} & 1 \\
1 & \frac{1}{V} \operatorname{var}\left\{\boldsymbol{\phi}_{n}^{\top} \mathbf{s}_{m}\right\}
\end{array}\right] .
$$

${ }^{1}$ Under the Gaussian $\mathrm{SCV}$ data-model assumption, $E\left\{\phi \phi^{\top}\right\}=E\left\{\mathbf{R}^{-1} \mathbf{s s}^{\top} \mathbf{R}^{-1}\right\}=\mathbf{R}^{-1}$. 
Two particular subcases in ICA are of interest. The first case is when the samples are iid with unit variance, for which $\mathbf{R}_{n}=\mathbf{I}_{V}, \boldsymbol{\Gamma}_{m}=E\left\{\phi_{m}^{2}\right\} \mathbf{I}_{V}$, and $\mathcal{K}_{m, n}=\kappa_{m}$, where $\kappa_{m} \triangleq E\left\{\phi_{m}^{2}\right\} \geq 1$. These simplifications give the same results as in [29. Eq. 38] and [30. Thm. 2], namely:

$$
\operatorname{ISR}_{m, n} \geq \frac{1}{V}\left(\kappa_{m}-\kappa_{n}^{-1}\right)^{-1}=\frac{1}{V} \frac{\kappa_{n}}{\kappa_{m} \kappa_{n}-1} .
$$

The second subcase of ICA is for sources with Gaussian sample-to-sample dependence, i.e., $\mathbf{s}_{\mathbf{n}} \sim \mathcal{N}\left(\mathbf{0}, \mathbf{R}_{n} \in \mathbb{R}^{V \times V}\right)$. Then we have that $\boldsymbol{\Gamma}_{m}=\mathbf{R}_{m}^{-1}$ and $\mathcal{K}_{m, n}=V^{-1} \operatorname{tr}\left(\mathbf{R}_{m}^{-1} \mathbf{R}_{n}\right)$, which corresponds to [31, Eq. 19].

\section{EXamples of Algorithm Performance ANd CRLB}

In this section, we compare the performance of several IVA algorithms versus the iCRLB given in Section VIII]

\section{A. MPE IVA}

For our first set of experiments, we consider sources following the multivariate power exponential (MPE) distribution, an elliptical distribution with $h_{e}(u)=\exp \left(-\frac{1}{2} u^{\beta}\right)$ and normalization constant $c_{K}=\pi^{-K / 2} 2^{-K /(2 \beta)} \beta \Gamma(K / 2)$, where $\beta>0$ is termed the shape parameter. This distribution possesses a score function which includes the score functions used in both [36] and [10] as special cases. In this section, we consider IVA with multivariate power exponential distribution model (IVA-MPE), where the algorithm was presented in [37], using simulated datasets with iid samples from the MPE family. The performance of IVA-MPE is compared with the iCRLB derived in Section VIII.

For this experiment, there are $N=3 \mathrm{MPE} \mid \mathrm{SCVS}$ of dimension $K=5$. All the sources use the same shape parameter, $\beta$. The covariance matrix associated with each source is randomly picked for the experiment, yet fixed for all trials in the experiment. The $k$ th entry of each SCV is used as a latent source for the $k$ th dataset. Entries of the random mixing matrices, $\mathbf{A}^{[k]}$, are from the standard normal distribution and are randomly selected for each trial.

We compute the theoretical iCRLB for ISR and compare this value with the ISR achieved using IVA-MPE with the correct shape parameter for each source. We then compute the total theoretical normalized ISR, defined as,

$$
\mathrm{ISR} \triangleq \sum_{m=1, n=1, m \neq n}^{N} V \operatorname{ISR}_{m, n} .
$$

We compare this theoretical ISR with the average ISR computed from 1000 independent trials of the algorithm as we vary the number of samples per dataset, $V$.

Due to the presence of local minima in the IVA objective function for non-Gaussian sources [38], the algorithm may converge to local minima. At local minima, the sources are separated within a dataset but the SCVs are not successfully identified, i.e., the permutation ambiguity is unresolved. We first compare the ICRLB for the ISR with the mean of the ISR achieved over successful trials. A trial is deemed successful if the location of the maximum absolute entry in each row of $\mathbf{G}^{[k]}=\mathbf{W}^{[k]} \mathbf{A}^{[k]}$ is unique within each dataset and colocated across the datasets (the former indicates sources are separated within each dataset and the latter indicates if the permutation ambiguity is resolved). The fraction of trials which are successful increases as $\beta$ decreases and/or as the sample size per dataset increases. The lowest success rate was $98 \%$, when $V=100$ and $\beta=6$. For all other settings the success rate was greater than 99.5\%. From Fig. 1. the performance of the IVA algorithm approaches the iCRLB as the sample size per dataset increases.

We also show in Fig. 2 -for the same experiment described above-the performance of the IVA-MPE when the algorithm selects between one of two shape parameters $(\beta \in\{0.5,2.0\})$ according to which shape parameter provides the lowest cost.

In another experiment, we use the same parameters as before except now the SCVs each have identity covariance matrices. For this experiment, there are nonidentifiable conditions as $\beta \rightarrow 1$, thus we compare the ICRLB for the ISR with the median rather than the mean. From Fig. 3, the performance of the IVA algorithm approaches the iCRLB as the sample size per dataset increases.

In both Fig. 1 and Fig. 3, the iCRLB follows the behavior predicted by Theorems 3 , 6, and 7 Namely, the iCRLB is infinite when sources are Gaussian and $\mathbf{R}_{n}=\mathbf{I}$ for all sources; the maximum ISR occurs when sources are Gaussian $(\beta=1)$; and as $\beta$ moves 'away' from one the non-Gaussianity measure $\kappa$ increases, which yields better source separation, i.e., lower ISR

\section{B. Orthogonal Generalized Joint Diagonalization with Second-Order Lags}

In this section, we consider the effect of sample dependency. To the best of our knowledge, there is only one algorithm in the IVA framework that accounts for sample-to-sample dependence, namely joint diagonalization via second-order statistics (JDIAG-SOS)] as given in [12]. The performance of JDIAG-SOS is compared with the iCRLB derived in Section VIII]

All the sources are a vector moving average of iid Gaussian samples, i.e.,

$$
\mathbf{s}_{n}(v)=\sum_{l=0}^{L-1} \mathbf{B}_{l} \mathbf{z}(v-l),
$$




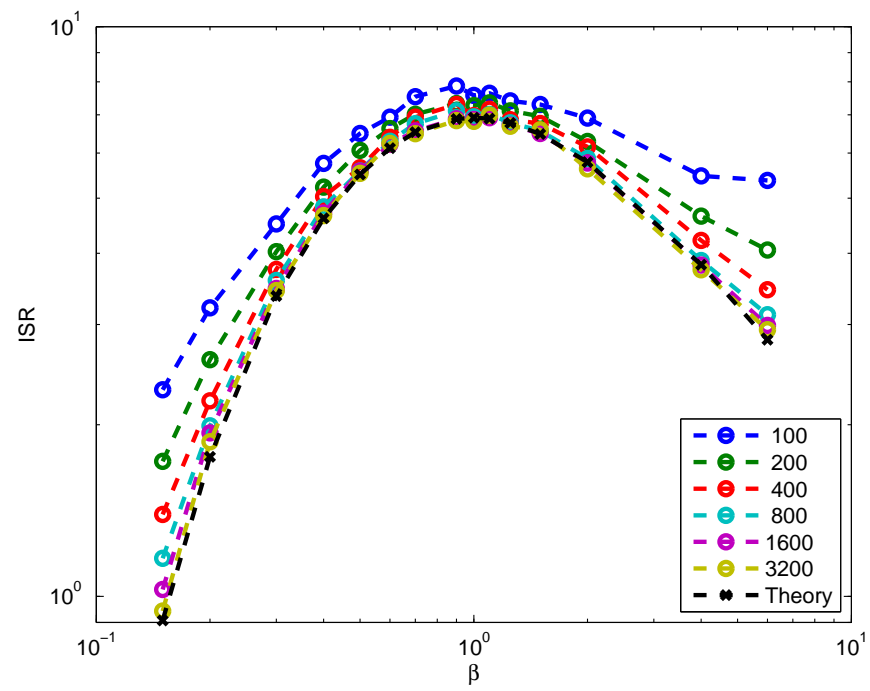

Fig. 1. The average ISR (of the successful trials) of IVA-MPE algorithm for various numbers of iid samples versus the shape parameter of the simulated SCV in the iid IVA experiment. The algorithm uses exact knowledge of the shape parameter. All results are compared with the iCRLB.

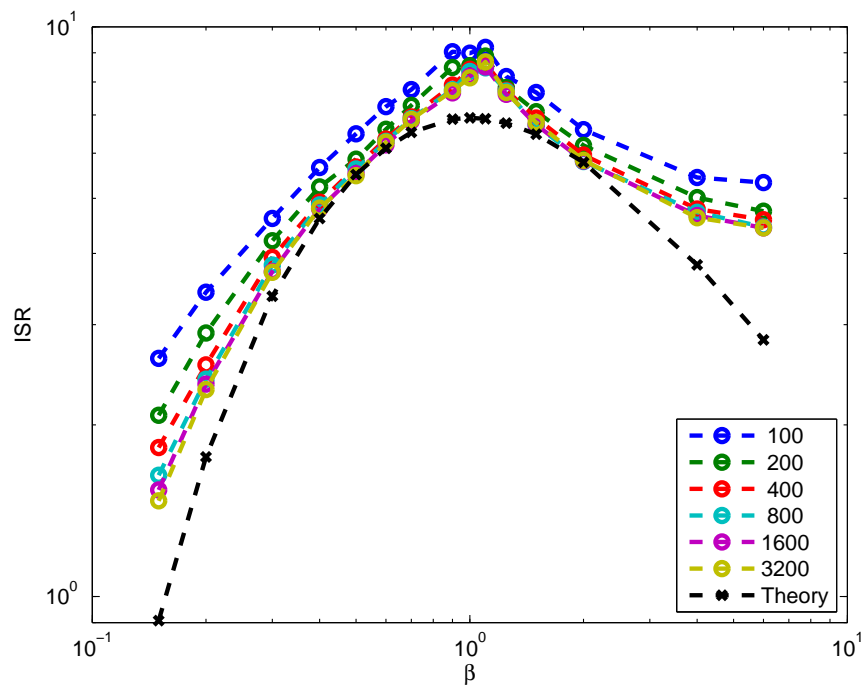

Fig. 2. The average ISR (of the successful trials) of IVA-MPE algorithm for various numbers of iid samples versus the shape parameter of the simulated SCV in the iid IVA experiment. The algorithm selects from one of two shape parameters, $\beta \in\{0.5,2.0\}$, and thus does not use exact knowledge of the shape parameter. All results are compared with the iCRLB.

where $\mathbf{z} \sim \mathcal{N}\left(\mathbf{0}, \mathbf{I}_{K}\right)$ and $\left[\mathbf{B}_{l}\right]_{k_{1}, k_{2}} \sim \mathcal{N}(0,1)$. For this experiment, there are $N=3$ sources for $K=3$ datasets, each with $V=1000$ samples and $L=4$. Entries of the random mixing matrices, $\mathbf{A}^{[k]}$, are from the standard normal distribution and are randomly selected for each trial.

We compute the theoretical $\mathrm{ICRLB}$ for $\mathrm{ISR}$ assuming the data was generated with $L=1, \ldots, 4$. Since $L=4$ for the data, the performance bound is shown to decrease until the lag is 3 . The performance bound for $L=4$ is shown for lags greater than 3. We compare the performance bounds with the average over 100 independent trials of the ISR achieved using JDIAG-SOS with various lags. Due to JDIAG-SOS estimating orthogonal demixing matrices there exists a noticeable difference between the iCRLB for ISR and the observed ISR

\section{CONClusion}

The use of IVA for the separation of multiple datasets concurrently has been a more recent development within the general BSS literature. A variety of algorithms have been developed that are essentially the multivariate extensions of [CA algorithms which take into account the dependence of sources between datasets in a variety of ways. There are three principal reasons for using these algorithms (versus just using ICA individually on each dataset). First, to increase the set of sources which can be identified. Second, to automatically 'align' dependent sources. Third, to maximize the achievable source separation. In this work, we have given the larger set of sources which can be identified by IVA, proven when the estimated sources can 


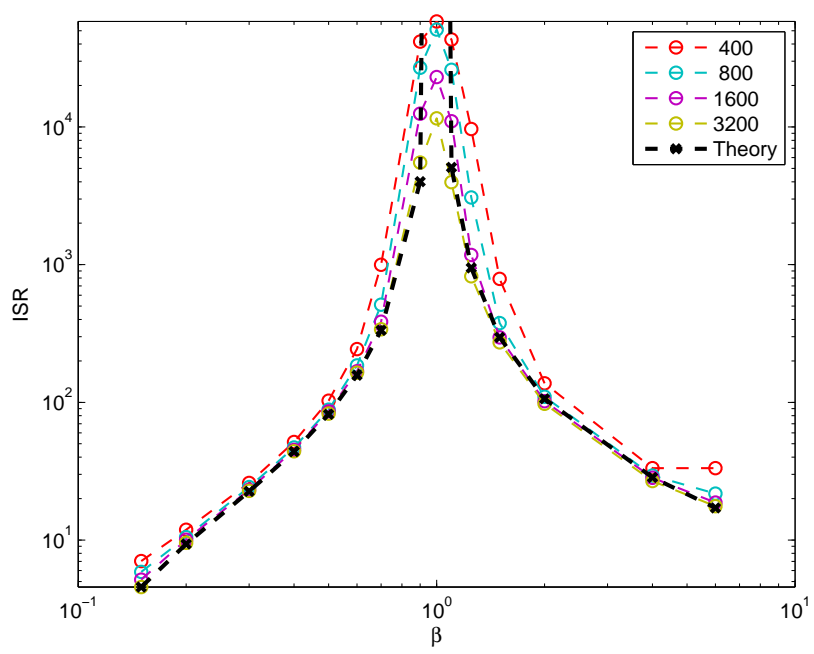

Fig. 3. The iCRLB theory for ISR as the shape parameter, $\beta$, varies is compared with the median ISR of all 1000 trials for different numbers of iid samples, $V$.

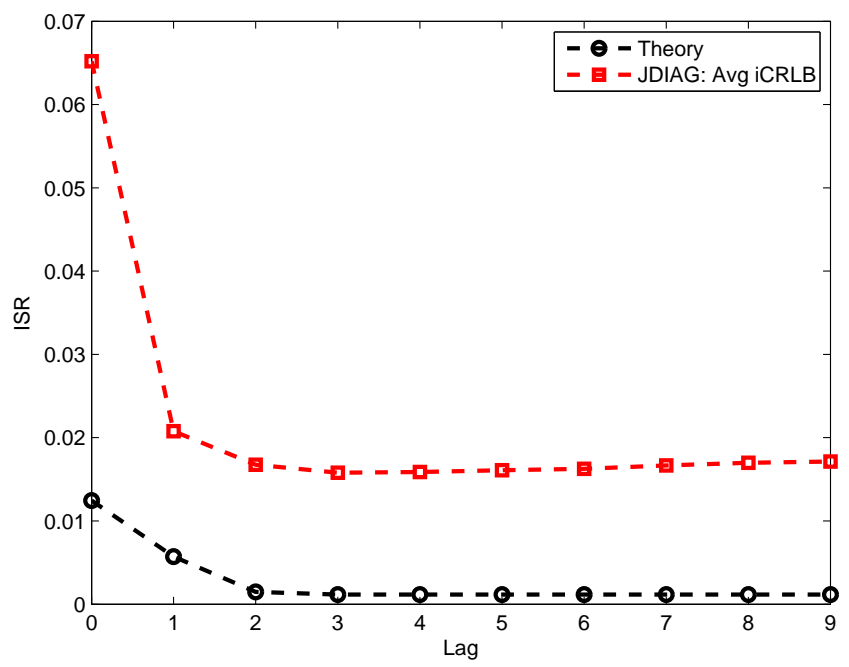

Fig. 4. The average ISR for 100 trials by JDIAG-SOS(L). The number of lags used by JDIAG-SOS is varied from 0 to $9(L=1, \ldots, 10)$. The iCRLB is shown assuming at most lag $=3$.

be 'aligned', and provided the bound on achievable source separation using IVA These results are achieved for an IVA that accounts for linear and nonlinear dependence of sources across datasets, non-Gaussianity, and sample-to-sample dependence. It is clear that IVA bridges the gap between CCA and ICA.

It will be interesting for future work to consider the additional diversity of complex-valued sources which are improper or noncircular. Additionally, our work will be useful for assessing the performance of future algorithms which account for sample dependency in an IVA framework.

\section{APPENDIX A}

\section{DERIVATION OF IVA FIM}

Here we derive the FIM of 11 wrt $\mathcal{W}$. The $K N^{2}$ parameters result in $K N^{2} \times K N^{2}$ dimension FIM with the entry associated with $w_{m_{1}, n_{1}}^{\left[k_{1}\right]}$ and $w_{m_{2}, n_{2}}^{\left[k_{2}\right]}$ given by (4).

For the computations to follow it is useful to observe that,

$$
\begin{gathered}
\frac{\partial \log \left|\operatorname{det} \mathbf{W}^{[l]}\right|}{\partial w_{m, n}^{[k]}}=\delta_{l, k} \mathbf{e}_{m}^{\top}\left(\mathbf{W}^{[k]}\right)^{-\top} \mathbf{e}_{n} \\
\mathbf{Y}_{m}^{\top}=\left[\left(\mathbf{X}^{[1]}\right)^{\top} \mathbf{w}_{m}^{[1]}, \ldots,\left(\mathbf{X}^{[K]}\right)^{\top} \mathbf{w}_{m}^{[K]}\right] \in \mathbb{R}^{K \times V},
\end{gathered}
$$




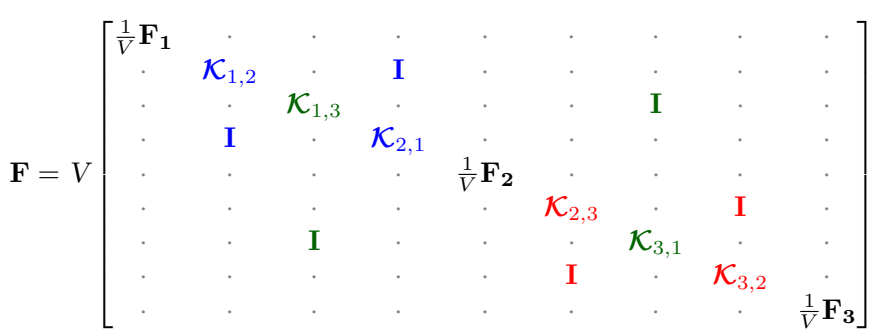

Fig. 5. Form of FIM when $N=3$ sources. All entries are of $K \times K$ matrices and we use $\cdot$ denote zero blocks. The entries of FIM associated with $\mathbf{F}_{1,2}$, $\mathbf{F}_{1,3}$, and $\mathbf{F}_{2,3}$ are indicated by blue, green, and red, respectively.

$$
\frac{\partial \mathbf{Y}_{l}}{\partial w_{m, n}^{[k]}}=\delta_{l, m} \operatorname{Diag}\left(\mathbf{e}_{k}\right) \mathbf{X}_{n} \in \mathbb{R}^{K \times V}
$$

and

$$
\begin{aligned}
\frac{\partial \log \left(p_{m}\left(\mathbf{Y}_{m}\right)\right)}{\partial w_{m, n}^{[k]}} & =\operatorname{tr}\left(\frac{\partial \log \left(p_{m}\left(\mathbf{Y}_{m}\right)\right)}{\partial \mathbf{Y}_{m}^{\top}} \frac{\partial \mathbf{Y}_{m}}{\partial w_{m, n}^{[k]}}\right) \\
& =-\operatorname{tr}\left(\mathbf{\Phi}_{m}^{\top} \operatorname{Diag}\left(\mathbf{e}_{k}\right) \mathbf{X}_{n}\right) \\
& =-\left(\boldsymbol{\phi}_{m}^{[k]}\right)^{\top} \mathbf{x}_{n}^{[k]} .
\end{aligned}
$$

Note that 36 is due to applying the chain rule given in [39, Sect. 2.8.1]. Thus the gradient of the likelihood function in (1) is

$$
\frac{\partial \mathcal{L}(\mathcal{W})}{\partial w_{m, n}^{[k]}}=-\left(\phi_{m}^{[k]}\right)^{\top} \mathbf{x}_{n}^{[k]}+V w_{n, m}^{-[k]}
$$

where $w_{m, n}^{-[k]}$ is the entry in the $m$ th row and $n$th column $\left(\mathbf{W}^{[k]}\right)^{-1}$.

Letting $\mathbf{A}=\mathbf{W}=\mathbf{I}$ we have the FIM of interest with entries given by

$$
\begin{aligned}
{[\mathbf{F}]_{k_{2}, m_{2}, n_{2}}^{k_{1}, m_{1}, n_{1}} \triangleq } & {\left.[\mathbf{F}(\mathcal{W})]_{k_{2}, m_{2}, n_{2}}^{k_{1}, m_{1}, n_{1}}\right|_{\mathbf{A}=\mathbf{I}, \mathbf{W}=\mathbf{I}} } \\
= & E\left\{\left(\left(\boldsymbol{\phi}_{m_{1}}^{\left[k_{1}\right]}\right)^{\top} \mathbf{s}_{n_{1}}^{\left[k_{1}\right]}\left(\mathbf{s}_{n_{2}}^{\left[k_{2}\right]}\right)^{\top} \boldsymbol{\phi}_{m_{2}}^{\left[k_{2}\right]}\right\}+V^{2} \delta_{m_{1}, n_{1}} \delta_{m_{2}, n_{2}}\right. \\
& -V E\left\{\left(\boldsymbol{\phi}_{m_{2}}^{\left[k_{2}\right]}\right)^{\top} \mathbf{s}_{n_{2}}^{\left[k_{2}\right]}\right\} \delta_{m_{1}, n_{1}} \\
& -V E\left\{\left(\boldsymbol{\phi}_{m_{1}}^{\left[k_{1}\right]}\right)^{\top} \mathbf{s}_{n_{1}}^{\left[k_{1}\right]}\right\} \delta_{m_{2}, n_{2}} \\
= & E\left\{\left(\phi_{m_{1}}^{\left[k_{1}\right]}\right)^{\top} \mathbf{s}_{n_{1}}^{\left[k_{1}\right]}\left(\mathbf{s}_{n_{2}}^{\left[k_{2}\right]}\right)^{\top} \boldsymbol{\phi}_{m_{2}}^{\left[k_{2}\right]}\right\}-V^{2} \delta_{m_{1}, n_{1}} \delta_{m_{2}, n_{2}},
\end{aligned}
$$

where the following expression holds, $E\left\{\mathbf{s}_{n}^{\left[k_{1}\right]}\left(\phi_{m}^{\left[k_{2}\right]}\right)^{\top}\right\}=\delta_{k_{1}, k_{2}} \delta_{m, n} \mathbf{I}_{V}$, see [2]. Since, by assumption, both $E\left\{\boldsymbol{\phi}_{m}^{[k]}\right\}=\mathbf{0}$ and $E\left\{\mathbf{s}_{m}^{[k]}\right\}=\mathbf{0}$, then it is true that $[\mathbf{F}]_{k_{2}, m_{2}, n_{2}}^{k_{1}, m_{1}, n_{1}}=0$ when one of the entries in $\left(m_{1}, n_{1}, m_{2}, n_{2}\right)$ is unique. It is also zero when $m_{1}=n_{1} \neq m_{2}=n_{2}$, i.e., $E\left\{\left(\phi_{m_{1}}^{\left[k_{1}\right]}\right)^{\top} \mathbf{s}_{m_{1}}^{\left[k_{1}\right]}\right\} E\left\{\left(\mathbf{s}_{m_{2}}^{\left[k_{2}\right]}\right)^{\top} \phi_{m_{2}}^{\left[k_{2}\right]}\right\}-V^{2}=V^{2}-V^{2}=0$. Thus, there are only three nonzero cases to consider:

$$
[\mathbf{F}]_{k_{2}, m_{2}, n_{2}}^{k_{1}, m_{1}, n_{1}}= \begin{cases}V\left(\mathcal{K}_{m_{1}, m_{1}}^{\left[k_{1}, k_{2}\right]}-V\right) & m_{1}=n_{2}=m_{2}=n_{1} \\ V \mathcal{K}_{m_{1}, n_{1}}^{\left[k_{1}, k_{2}\right]} & m_{1}=m_{2} \neq n_{1}=n_{2} \\ V \delta_{k_{1}, k_{2}} & m_{1}=n_{2} \neq m_{2}=n_{1} \\ 0 & \text { otherwise, }\end{cases}
$$

where $\mathcal{K}_{m, n}^{\left[k_{1}, k_{2}\right]} \triangleq \frac{1}{V} E\left\{\left(\phi_{m}^{\left[k_{1}\right]}\right)^{\top} \mathbf{s}_{n}^{\left[k_{1}\right]}\left(\mathbf{s}_{n}^{\left[k_{2}\right]}\right)^{\top} \phi_{m}^{\left[k_{2}\right]}\right\}=\frac{1}{V} \operatorname{tr}\left(E\left\{\phi_{m}^{\left[k_{2}\right]}\left(\phi_{m}^{\left[k_{1}\right]}\right)^{\top} \mathbf{s}_{n}^{\left[k_{1}\right]}\left(\mathbf{s}_{n}^{\left[k_{2}\right]}\right)^{\top}\right\}\right)$ is the $\left(k_{1}, k_{2}\right)$ entry of $\mathcal{K}_{m, n}$. The form of this matrix (e.g., see Fig. 5p is the block-matrix extension of that for the single dataset FIM] given in [32]. 
There exists a permuted FIM in which there are $N+N(N-1) / 2$ nonzero matrices along the diagonal, i.e.,

$$
\mathbf{F}=\left[\begin{array}{cc}
\oplus_{n=1}^{N} \mathbf{F}_{n} & \mathbf{0} \\
\mathbf{0} & \oplus_{m=1, n=m+1}^{N, N} \mathbf{F}_{m, n}
\end{array}\right]
$$

The submatrices are given by

$$
\mathbf{F}_{n} \triangleq \operatorname{var}\left\{\operatorname{diag}\left(\mathbf{\Phi}_{n} \mathbf{S}_{n}^{\top}-\mathbf{I}_{V}\right)\right\}=V\left(\mathcal{K}_{n, n}-V \mathbf{1}_{K \times K}\right)
$$

and

$$
\mathbf{F}_{m, n} \triangleq \operatorname{cov}\left\{\left[\begin{array}{c}
\operatorname{diag}\left(\mathbf{\Phi}_{m} \mathbf{S}_{n}^{\top}\right) \\
\operatorname{diag}\left(\mathbf{\Phi}_{n} \mathbf{S}_{m}^{\top}\right)
\end{array}\right]\right\}=V\left[\begin{array}{cc}
\mathcal{K}_{m, n} & \mathbf{I}_{K} \\
\mathbf{I}_{K} & \mathcal{K}_{n, m}
\end{array}\right]
$$

where $\mathbf{F}_{n} \in \mathbb{R}^{K \times K}$ and $\mathbf{F}_{m, n} \in \mathbb{R}^{2 K \times 2 K}$. It is also useful to note that for $1 \leq m \neq n \leq N$ we have $\mathcal{K}_{m, n}^{\left[k_{1}, k_{2}\right]}=$ $\frac{1}{V} \operatorname{tr}\left(\boldsymbol{\Gamma}_{m}^{\left[k_{2}, k_{1}\right]} \mathbf{R}_{n}^{\left[k_{1}, k_{2}\right]}\right)$, where $\mathbf{R}_{n}^{\left[k_{1}, k_{2}\right]} \triangleq E\left\{\mathbf{s}_{n}^{\left[k_{1}\right]}\left(\mathbf{s}_{n}^{\left[k_{2}\right]}\right)^{\top}\right\} \in \mathbb{R}^{V \times V}$ and $\boldsymbol{\Gamma}_{n}^{\left[k_{1}, k_{2}\right]} \triangleq E\left\{\boldsymbol{\phi}_{n}^{\left[k_{1}\right]}\left(\boldsymbol{\phi}_{n}^{\left[k_{2}\right]}\right)^{\top}\right\} \in \mathbb{R}^{V \times V}$.

\section{APPENDIX B}

\section{Score Function Covariance Matrix fOr Elliptical Distributions}

In this appendix, we show that the score function covariance matrix, $\Gamma=E\left\{\phi \phi^{\top}\right\}$, is a scalar multiple of the inverse of the covariance matrix for all elliptical distributions defined by $(29)$. We begin by letting $\mathbf{z}=\mathbf{\Sigma}^{-1 / 2} \mathbf{x}$ so that $p_{\mathbf{z}}(\mathbf{z})=$ $\left|\operatorname{det} \boldsymbol{\Sigma}^{-1 / 2}\right|^{-1} p_{\mathbf{x}}\left(\boldsymbol{\Sigma}^{1 / 2} \mathbf{z}\right)=c_{K} h_{e}\left(\mathbf{z}^{\top} \mathbf{z}\right)$, which results in $\boldsymbol{\Gamma}=\boldsymbol{\Sigma}^{-1 / 2} E\left\{g^{2}\left(\mathbf{z}^{\top} \mathbf{z}\right) \mathbf{z z}^{\top}\right\} \boldsymbol{\Sigma}^{-1 / 2}$. To compute the expectation requires the following multivariate integral to be evaluated:

$$
E\left\{g^{2}\left(\mathbf{z}^{\top} \mathbf{z}\right) z_{l} z_{k}\right\}=\int_{-\infty}^{\infty} g^{2}\left(\mathbf{z}^{\top} \mathbf{z}\right) z_{l} z_{k} p(\mathbf{z}) d \mathbf{z} .
$$

We use a transformation of variables utilized for similar problems in [40], [41], namely,

$$
\begin{aligned}
z_{1} & =r \prod_{k=1}^{K-1} \sin \theta_{k} \\
z_{j} & =r\left(\prod_{k=1}^{K-j} \sin \theta_{k}\right) \cos \theta_{K-j+1}, 2 \leq j \leq K-1 \\
z_{K} & =r \cos \theta_{1}
\end{aligned}
$$

where $0<\theta_{j} \leq \pi, j=1, \ldots, K-2,0<\theta_{K-1} \leq 2 \pi, 0<r \leq \infty$. By noting that $\mathbf{z}^{\top} \mathbf{z}=r^{2}$ and the Jacobian of the transformation from $\mathbf{z}$ to $\left[\theta_{1} \ldots \theta_{K-1} r\right]^{\top}$ is $r^{K-1} \sin ^{K-2} \theta_{1} \sin ^{K-3} \theta_{2} \cdots \sin \theta_{K-2}=r^{K-1} \prod_{k=1}^{K-2}\left(\sin \theta_{k}\right)^{K-1-k}$, we have $p(r)=c_{K} h_{e}\left(r^{2}\right)$.

There are two cases, $l=k$ and $l \neq k$, required to evaluate (43). Let us consider the former first,

$$
E\left\{g^{2}\left(\mathbf{z}^{\top} \mathbf{z}\right) z_{1}^{2}\right\}=E\left\{g^{2}\left(r^{2}\right) r^{K+1}\right\} \frac{2 \pi^{K / 2}}{K \Gamma(K / 2)},
$$

where we have made use of $\int_{0}^{\pi} \sin ^{n} \theta d \theta=\sqrt{\pi} \Gamma[(n+1) / 2] / \Gamma[(n+2) / 2]$ when $n \geq 1$.

Now for the off-diagonal terms, e.g., when $K=2, E\left\{g^{2}\left(\mathbf{z}^{\mathbf{\top}} \mathbf{z}\right) z_{1} z_{2}\right\}=\int_{0}^{\infty} \int_{0}^{2 \pi} g^{2}\left(r^{2}\right) p(r) r \cos \theta \sin \theta d \theta d r=0$, where we have used the following $\int_{0}^{n \pi} \cos (\theta) \sin ^{n}(\theta) d \theta=0$ when $n \in \mathbb{N}^{*}$. The result holds for the more general case when $K>2$ and $l \neq k$ and we arrive at the final expression of $E\left\{\phi \phi^{\top}\right\}=\boldsymbol{\Sigma}^{-1 / 2} E\left\{g^{2}\left(\mathbf{z}^{\top} \mathbf{z}\right) \mathbf{z} \mathbf{z}^{\top}\right\} \boldsymbol{\Sigma}^{-1 / 2}=E\left\{g^{2}\left(\mathbf{z}^{\top} \mathbf{z}\right) \mathbf{z z}^{\top}\right\} \mathbf{\Sigma}^{-1}=$ $\kappa \mathbf{R}^{-1}, K \geq 2$, where $\kappa \triangleq E\left\{g^{2}\left(r^{2}\right) r^{K+1}\right\} \frac{2 \pi^{K / 2}}{K \Gamma(K / 2)} \rho$.

\section{REFERENCES}

[1] A. Hyvärinen, J. Karhunen, and E. Oja, Independent Component Analysis. Wiley-Interscience, 2001.

[2] P. Comon and C. Jutten, Handbook of Blind Source Separation: Independent Component Analysis and Applications, 1st ed. Academic Press, 2010.

[3] J.-H. Lee, T.-W. Lee, F. A. Jolesz, and S.-S. Yoo, "Independent vector analysis (IVA): Multivariate approach for fMRI group study," NeuroImage, vol. 40, no. 1, pp. 86-109, 2008.

[4] Y.-O. Li, T. Adali, W. Wang, and V. D. Calhoun, "Joint blind source separation by multiset canonical correlation analysis," IEEE Trans. Signal Process., vol. 57, no. 10, pp. 3918-3929, Oct. 2009.

[5] T. Kim, "Real-time independent vector analysis for convolutive blind source separation," Circuits and Systems I: Regular Papers, IEEE Transactions on, vol. 57, no. 7, pp. 1431-1438, Jul. 2010.

[6] J. R. Kettenring, "Canonical analysis of several sets of variables," Biometrika, vol. 58, no. 3, pp. 433-451, 1971.

[7] F. W. Young, J. De Leeuw, and Y. Takane, "Regression with qualitative and quantitative variables: An alternating least squares method with optimal scaling features," Psychometrika, vol. 41, pp. 505-529, 1976. 
[8] M. Anderson, T. Adal, and X.-L. Li, "Joint blind source separation of multivariate Gaussian sources: Algorithms and performance analysis," IEEE Trans. Signal Process., vol. 60, no. 4, pp. 1672-1683, Apr. 2012.

[9] H. Hotelling, "Relations between two sets of variates," Biometrika, vol. 28, no. 3/4, pp. 321-377, 1936.

[10] M. Anderson, X.-L. Li, and T. Adal1, "Nonorthogonal independent vector analysis using multivariate Gaussian model," in Latent Variable Analysis and Signal Separation, ser. Lecture Notes in Computer Science. Springer Berlin / Heidelberg, 2010, vol. 6365, pp. 354-361.

[11] J. Vía, M. Anderson, X.-L. Li, and T. Adall, "A maximum likelihood approach for independent vector analysis of Gaussian data sets," in IEEE International Workshop on Machine Learning for Signal Processing (MLSP 2011), Beijing, China, Sep. 2011.

[12] X.-L. Li, T. Adalı, and M. Anderson, "Joint blind source separation by generalized joint diagonalization of cumulant matrices," Signal Process., vol. 91, no. 10, pp. 2314-2322, Oct. 2011.

[13] X.-L. Li, M. Anderson, and T. Adal, "Second and higher-order correlation analysis of multiset multidimensional variables by joint diagonalization," in Latent Variable Analysis and Signal Separation, ser. Lecture Notes in Computer Science. Springer Berlin / Heidelberg, 2010, vol. 6365, pp. 197-204.

[14] J. de Leeuw, "The Gifi-system of nonlinear multivariate analysis," Data Analysis and Informatics, vol. III, pp. 415-424, 1984.

[15] A. Gifi, Nonlinear multivariate analysis. New York: Wiley, 1990.

[16] X. Yin, "Canonical correlation analysis based on information theory," Journal of Multivariate Analysis, vol. 91, no. 2, pp. 161-176, 2004.

[17] S. Akaho, "A kernel method for canonical correlation analysis," in International Meeting on Psychometric Society (IMPS2001), 2001.

[18] T. Melzer, M. Reiter, and H. Bischof, "Nonlinear feature extraction using generalized canonical correlation analysis," in Artificial Neural Networks ICANN 2001, ser. Lecture Notes in Computer Science, G. Dorffner, H. Bischof, and K. Hornik, Eds. Springer Berlin Heidelberg, 2001, vol. 2130, pp. $353-360$.

[19] K. Todros and A. O. Hero, "On measure transformed canonical correlation analysis," IEEE Trans. Signal Process., vol. 60, no. 9, pp. 4570-4585, Sep. 2012.

[20] T. Kim, T. Eltoft, and T.-W. Lee, "Independent vector analysis: an extension of ICA to multivariate components," in Independent Component Analysis and Blind Signal Separation, ser. Lecture Notes in Computer Science. Springer Berlin / Heidelberg, 2006, vol. 3889, pp. $165-172$.

[21] T. Kim, I. Lee, and T.-W. Lee, "Independent vector analysis: Definition and algorithms," in Proc. of 40th Asilomar Conference on Signals, Systems, and Computers, Oct. 2006, pp. 1393-1396.

[22] A. Hiroe, "Solution of permutation problem in frequency domain ICA, using multivariate probability density functions," in Independent Component Analysis and Blind Signal Separation, ser. Lecture Notes in Computer Science, J. Rosca, D. Erdogmus, J. C. Príncipe, and S. Haykin, Eds. Springer Berlin Heidelberg, 2006, vol. 3889, pp. 601-608.

[23] P. Smaragdis, "Blind separation of convolved mixtures in the frequency domain," Neurocomputing, vol. 22, no. 1-3, pp. 21-34, 1998.

[24] R. Phlypo, "Jacobi iterations for canonical dependence analysis," Signal Processing, vol. 93, no. 1, pp. 185-197, 2013.

[25] R. A. Horn and C. R. Johnson, Matrix Analysis. Cambridge: Cambridge University Press, 1985.

[26] T. Kim, H. T. Attias, S.-Y. Lee, and T.-W. Lee, "Blind source separation exploiting higher-order frequency dependencies," IEEE Trans. Audio Speech Lang. Process., vol. 15, no. 1, pp. 70-79, Jan. 2007.

[27] T. M. Cover and J. A. Thomas, Elements of Information Theory. Wiley-Interscience, 2006.

[28] J.-F. Cardoso and B. H. Laheld, "Equivariant adaptive source separation," IEEE Trans. Signal Process., vol. 44, no. 12, pp. 3017-3030, Dec. 1996.

[29] P. Tichavský, Z. Koldovský, and E. Oja, "Performance analysis of the FastICA algorithm and Cramér-Rao bounds for linear independent component analysis," IEEE Trans. Signal Process., vol. 54, no. 4, pp. 1189-1203, Apr. 2006.

[30] E. Ollila, K. Hyon-Jung, and V. Koivunen, "Compact Cramér-Rao bound expression for independent component analysis," IEEE Trans. Signal Process., vol. 56, no. 4, pp. 1421-1428, Apr. 2008.

[31] A. Yeredor, "Blind separation of Gaussian sources with general covariance structures: Bounds and optimal estimation," IEEE Trans. Signal Process., vol. 58, no. 10 , pp. 5057-5068, Oct. 2010.

[32] B. Loesch and B. Yang, "Cramer-rao bound for circular and noncircular complex independent component analysis," IEEE Trans. Signal Process., vol. 61, no. 2, pp. 365-379, Jan. 2013.

[33] B. Afsari, "Sensitivity analysis for the problem of matrix joint diagonalization," SIAM J. Matrix Anal. Appl., vol. 30, no. 3, pp. 1148-1171, Sep. 2008.

[34] P. Comon, "Independent component analysis, a new concept?" Signal Process., vol. 36, no. 3, pp. 287-314, 1994.

[35] P. Lavergne, "A Cauchy-Schwarz inequality for expectation of matrices," Department of Economics, Simon Fraser University, Discussion Papers, 2008.

[36] T. Kim, "Independent vector analysis," Ph.D. dissertation, Department of BioSystems Korea Advanced Institute of Science and Technology, 2006.

[37] M. Anderson, G.-S. Fu, R. Phlypo, and T. Adalı, "Independent vector analysis, the Kotz distribution, and performance bounds," in Proc. IEEE Int. Conf. Acoust., Speech Signal Process. (ICASSP), 2013, accepted.

[38] M. Davies, "Audio source separation," Math. Signal Process. V, pp. 57-68, 2002.

[39] K. B. Petersen and M. S. Pedersen, "The matrix cookbook," Nov. 2008. [Online]. Available: http://matrixcookbook.com/

[40] R. J. Muirhead, Aspects of Multivariate Statistical Theory. Wiley-In, 2005.

[41] G. Aulogiaris and K. Zografos, "A maximum entropy characterization of symmetric Kotz type and Burr multivariate distributions," TEST, vol. 13, pp. 65-83, 2004, 10.1007/BF02603001. 